\title{
LWR First Recycle of TRU with Thorium Oxide for Transmutation and Cross Sections
}

Sonat Sen

Andrea Alfonsi

Gilles Youinou

February 2013

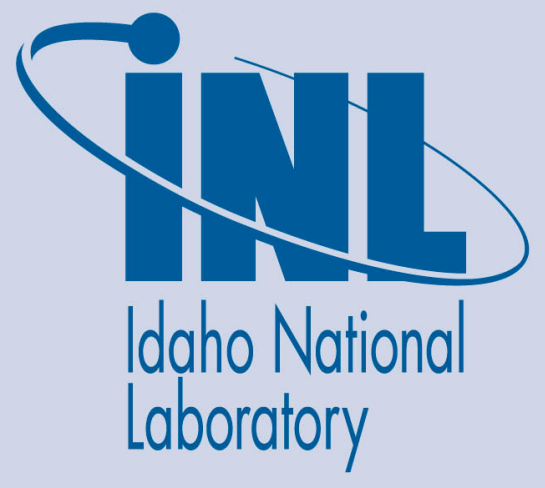

The INL is a U.S. Department of Energy National Laboratory operated by Battelle Energy Alliance 
INL/EXT-12-26633

Rev. 1

FCRD-FCT-2013-000103

\title{
LWR First Recycle of TRU with Thorium Oxide for Transmutation and Cross Sections
}

\author{
Sonat Sen \\ Andrea Alfonsi \\ Gilles Youinou
}

February 2013

Idaho National Laboratory
Idaho Falls, Idaho 83415

http://www.inl.gov

\author{
Prepared for the \\ U.S. Department of Energy \\ Office of Nuclear Energy \\ Under DOE Idaho Operations Office \\ Contract DE-AC07-05ID14517
}




\section{DISCLAIMER}

This information was prepared as an account of work sponsored by an agency of the U.S. Government. Neither the U.S. Government nor any agency thereof, nor any of their employees, makes any warranty, expressed or implied, or assumes any legal liability or responsibility for the accuracy, completeness, or usefulness, of any information, apparatus, product, or process disclosed, or represents that its use would not infringe privately owned rights. References herein to any specific commercial product, process, or service by trade name, trade mark, manufacturer, or otherwise, does not necessarily constitute or imply its endorsement, recommendation, or favoring by the U.S. Government or any agency thereof. The views and opinions of authors expressed herein do not necessarily state or reflect those of the U.S. Government or any agency thereof. 



\section{TABLE OF CONTENTS}

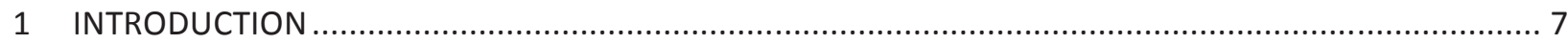

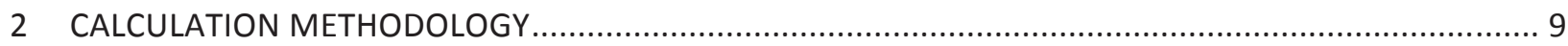

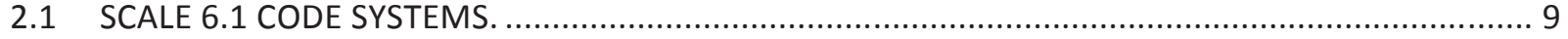

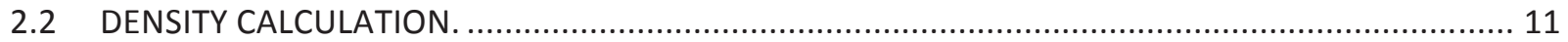

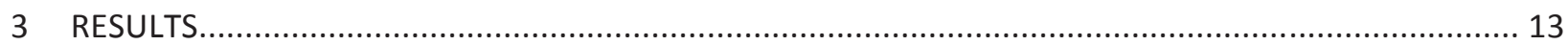

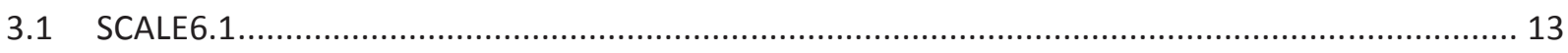

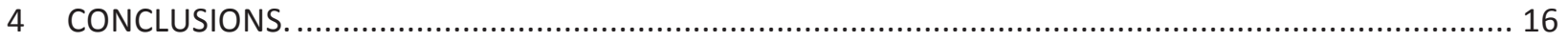

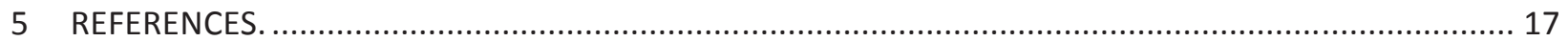

Appendix 1: Detailed isotopic mass fraction input (after 2-yrs fuel aging). ........................................ 18

Appendix 2: Detailed isotopic mass fraction output (discharged burn-up $50 \mathrm{GWd} / \mathrm{tHM}$ )................... 24

Appendix 3: Mid-cycle 1-group cross sections of most important actinides.......................................... 37 


\section{ACRONYMS}

$\begin{array}{ll}T h & \text { Thorium } \\ U & \text { Uranium } \\ A m & \text { Americium } \\ B k & \text { Berkelium } \\ C f & \text { Californium } \\ C m & \text { Curium } \\ F P & \text { Fission Product } \\ G W d & \text { Gigawatt-days } \\ I N L & \text { Idaho National Laboratory } \\ I t H M & \text { Initial tons of Heavy Metals } \\ L E U & \text { Low Enriched Uranium } \\ L W R & \text { Light Water Reactor } \\ M O X & \text { Mixed Oxide } \\ N p & \text { Neptunium } \\ P a & \text { Protactinium } \\ P u & \text { Plutonium } \\ T R U & \text { Transuranic } \\ U O X & \text { Uranium Oxide } \\ w / o & \text { weight percent }\end{array}$




\section{LWR FIRST RECYCLE OF TRU WITH THORIUM OXIDE FOR TRANSMUTATION AND CROSS SECTIONS}

\section{INTRODUCTION}

Thorium has been considered as an option to uranium-based fuel, based on considerations of resource utilization (thorium is approximately three times more plentiful than uranium) and as a result of concerns about proliferation and waste management (e.g. reduced production of plutonium, etc.). Since the average composition of natural Thorium is dominated (100\%) by the fertile isotope Th-232, Thorium is only useful as a resource for breeding new fissile materials, in this case U-233. Consequently a certain amount of fissile material must be present at the start-up of the reactor in order to guarantee its operation. The thorium fuel can be used in both oncethrough and recycle options, and in both fast and thermal spectrum systems.

The present study has been aimed by the necessity of investigating the option of using reprocessed plutonium/TRU, from a once-through reference LEU scenario (50 GWd/tHM), mixed with natural thorium and the need of collect data (mass fractions, cross-sections etc.) for this particular fuel cycle scenario. As previously pointed out, the fissile plutonium is needed to guarantee the operation of the reactor.

Four different scenarios have been considered:

- Thorium - recycled Plutonium;

- Thorium - recycled Plutonium/Neptunium;

- Thorium - recycled Plutonium/Neptunium/Americium;

- Thorium - recycled Transuranic.

The calculations have been performed with SCALE6.1-TRITON.

The amount of transuranic present in the reference $50.0 \mathrm{GWd} / \mathrm{tHM}$ UOX SNF (4.2\% U-235) after 5 years of cooling time is given in Table 1.1 below.

\begin{tabular}{|c|c|}
\hline Isotope & $\mathrm{kg} / \mathrm{tIHM}$ \\
\hline Np237 & $6.497413 \mathrm{E}-01$ \\
\hline Np238 & $1.550531 \mathrm{E}-10$ \\
\hline Np239 & $1.691246 \mathrm{E}-07$ \\
\hline $\mathrm{Np} 240 \mathrm{~m}$ & $4.848635 \mathrm{E}-18$ \\
\hline Pu236 & $2.000000 \mathrm{E}-97$ \\
\hline Pu238 & $3.160877 \mathrm{E}-01$ \\
\hline Pu239 & $6.175266 \mathrm{E}+00$ \\
\hline Pu240 & $2.945851 \mathrm{E}+00$ \\
\hline$\overline{\text { Pu241 }}$ & $1.406346 \mathrm{E}+00$ \\
\hline Pu242 & $8.728926 \mathrm{E}-01$ \\
\hline
\end{tabular}




\begin{tabular}{|c|c|}
\hline Pu243 & $3.494420 \mathrm{E}-16$ \\
\hline Pu244 & $2.893894 \mathrm{E}-05$ \\
\hline Pu246 & $1.799982 \mathrm{E}-21$ \\
\hline Am241 & $4.443372 \mathrm{E}-01$ \\
\hline Am242 & $9.896332 \mathrm{E}-09$ \\
\hline Am242M & $8.269964 \mathrm{E}-04$ \\
\hline Am243 & $1.968006 \mathrm{E}-01$ \\
\hline Am245 & $6.709985 \mathrm{E}-19$ \\
\hline Am246 & $9.216389 \mathrm{E}-25$ \\
\hline Cm242 & $1.308463 \mathrm{E}-05$ \\
\hline Cm243 & $6.755201 \mathrm{E}-04$ \\
\hline Cm244 & $6.995664 \mathrm{E}-02$ \\
\hline Cm245 & $5.596512 \mathrm{E}-03$ \\
\hline Cm246 & $7.152499 \mathrm{E}-04$ \\
\hline Cm247 & $9.800633 \mathrm{E}-06$ \\
\hline Cm248 & $7.574186 \mathrm{E}-07$ \\
\hline Cm250 & $4.286988 \mathrm{E}-15$ \\
\hline Bk249 & $1.728293 \mathrm{E}-10$ \\
\hline Bk250 & $1.267657 \mathrm{E}-23$ \\
\hline Cf249 & $1.039076 \mathrm{E}-08$ \\
\hline Cf250 & $1.554787 \mathrm{E}-09$ \\
\hline Cf251 & $9.700168 \mathrm{E}-10$ \\
\hline Cf252 & $1.750708 \mathrm{E}-10$ \\
\hline Total Pu & $1.171647 \mathrm{E}+01$ \\
\hline Total Np & $6.497414 \mathrm{E}-01$ \\
\hline Total Am & $6.419649 \mathrm{E}-01$ \\
\hline Total Cm & $7.696756 \mathrm{E}-02$ \\
\hline Total Bk & $1.728293 \mathrm{E}-10$ \\
\hline Total Cf & $1.309063 \mathrm{E}-08$ \\
\hline Total TRU & $1.308515 \mathrm{E}+01$ \\
\hline
\end{tabular}

Table 1.1 - TRU composition in the reference $50 \mathrm{GWd} / \mathrm{tHM}$ UOX SNF (5-year cooling time). 


\section{CALCULATION METHODOLOGY}

As previously said, the calculations needed by this study have been performed through the code SCALE 6.1/TRITON.

A small overview on the main capabilities and models used is reported in the following paragraphs.

\subsection{SCALE 6.1 CODE SYSTEMS.}

The reactor physics calculations have been performed by the lattice physics capabilities of the SCALE 6.1 code systems. The calculation flow consists of the use of several modules mutually coupled. The discrete-ordinates code NEWT (New ESC-based Weighting Transport code) coupled to the depletion code ORIGEN [8] via the TRITON control module [4]. Using the discrete-ordinates approximation to the transport equation on an arbitrary grid, together with a 238-group neutron cross-section library based on ENDF/B-VII, NEWT provides a robust and rigorous deterministic solution for non-orthogonal configurations. The differencing scheme employed by NEWT, the Extended Step Characteristic Approximation, allows a computational two-dimensional mesh based on arbitrary polygons. Such a mesh can be used to closely approximate curved or irregular surfaces to provide the capability to model problems that were formerly difficult or impractical to model directly with discrete-ordinates methods.

The TRITON control module performs the task of coordination of data transfer between various physics codes available within SCALE 6.1 and of invoking those codes in the proper sequence for a desired type of calculation. The high-fidelity nature of the NEWT solution in estimating angular flux distributions combined with the rigor of the ORIGEN depletion solver gives TRITON the capability to perform precise burnup-dependent physics calculations with few implicit approximations, and limited primarily by the accuracy of nuclide cross-sectional data. Such rigor may be necessary to capture the unique attributes of MOX/Th-TRU fuel behavior as well as that of advanced, highly heterogeneous fuel assembly designs being deployed in currentgeneration reactors. Cross-sectional self-shielding is carried out by BONAMI for unresolvedrange resonance data; the resolved resonance processor module CENTRM performs a 1-D discrete-ordinates code that uses point-wise cross-section data to produce a set of continuousenergy fluxes at discrete spatial intervals for each unit cell. Following a CENTRM calculation, the code PMC uses the resulting flux to collapse the point-wise continuous-energy cross sections into multi-group cross sections for each nuclide in each material in a unit (e.g., pin cell). The result is a multi-group library in which point cross-sectional data are weighted using the explicit point-wise spectrum representative of the nuclides present in a pin cell. Effects from overlapping resonances, fissile material in the fuel and surrounding moderator, anisotropic scattering, and inelastic level scattering are explicitly handled by this approach.

For the physics calculations carried out during this study, a TRITON model of one fourth standard (17x17) fuel assembly has been used (Fig 2.1). All the Th-TRU rods have the same Th232 enrichment and the same Plutonium content. The $0.5 \mathrm{~mm}$ water gap at the periphery is explicitly represented. The model uses three different burn-up zones to take into account the different local moderating ratios: 1 for the corner rods (green), 1 for the other rods located at the periphery (cyan) and 1 for the internal rods (red). 


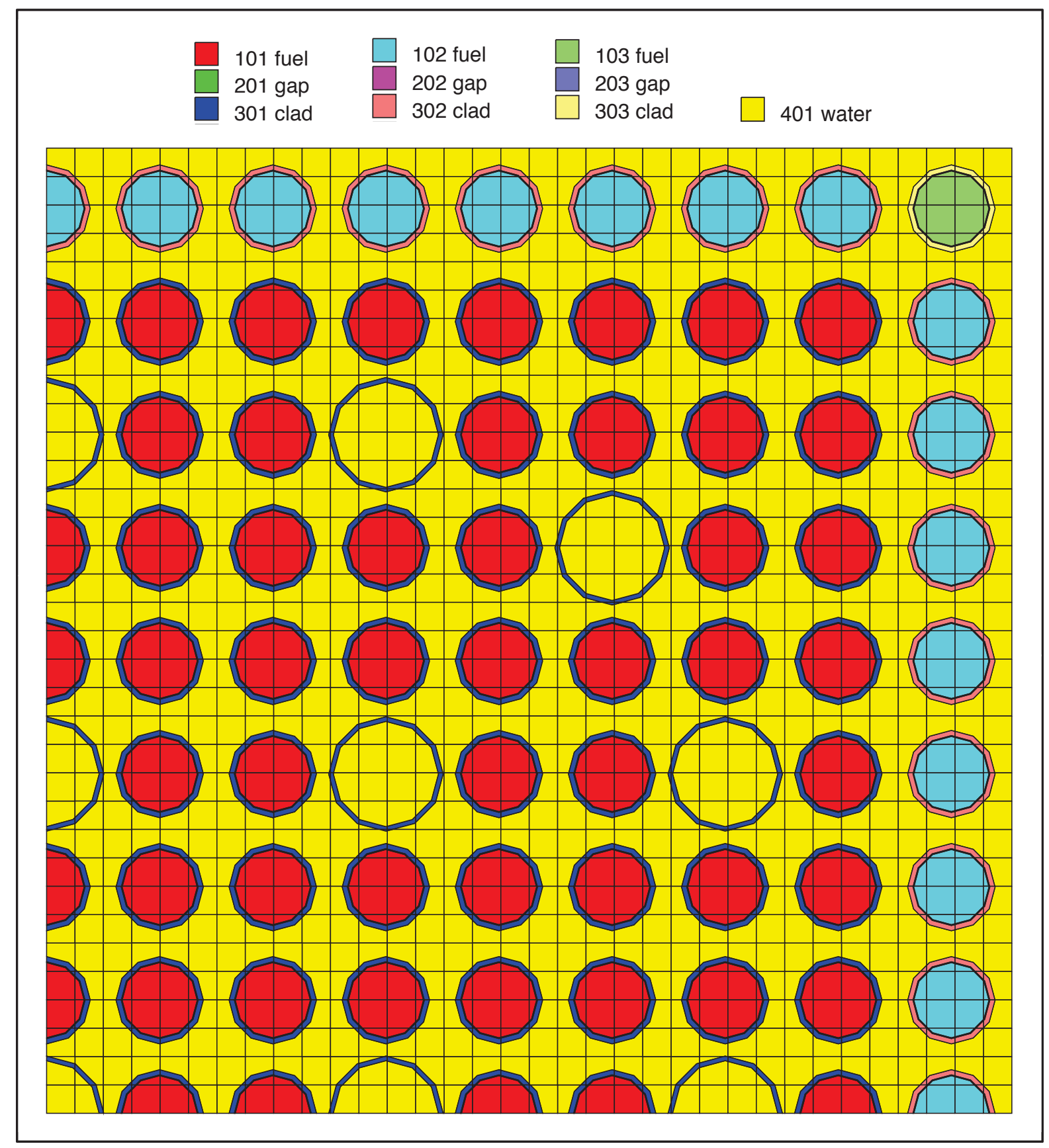

Figure 2.1 - TRITON model (one fourth of a standard 17x17 fuel assembly)

The TRU content in the Th-TRU fuel assemblies has been tuned to maintain the same average discharge burn-up and a constant cycle length. The calculations have been run considering a 3batch core with an average discharge burn-up of 50.0 GWd/tHM and a fuel power density of 39.2 MW/tHM. The model takes in account 2 year fuel aging (time needed by the fabrication and transport operations) and 5 years of cooling time (time needed in order to reduce the decay heat generation to levels compatible with the separation operations). The burn-up level above 
specified is reached after $\sim 1275$ days of irradiation. One cycle then corresponds to 2 years + $50 \mathrm{GWd} / \mathrm{tHM}$ (1275 days) + 5 years cooling time $\cong 10.5$ years.

The necessary $T R U^{1}$ enrichments are determined with a methodology that is standard for this kind of application: the k-infinity of the assembly at the average end of cycle burn-up (33.33 $\mathrm{GWd} / \mathrm{tHM}$ ), without soluble boron, is equal to that of the reference $4.2 \% \mathrm{UOX}$ assembly calculated using the same code (SCALE 6.1), methods (SN), nuclear data (238 group library based on ENDF/B - VII), etc., i.e. $\mathbf{k - i n f}=\mathbf{1 . 0 2 9 6 4 3}$ in our case. Figure 2-2, below, shows the kinfinity trends for all the cases considered in this study as a function of burnup in GWd/tHM. The red vertical line in Figure 2-2 corresponds to $33.33 \mathrm{GWd} / \mathrm{tHM}$, where k-infinity for all the cases are equal to each other.

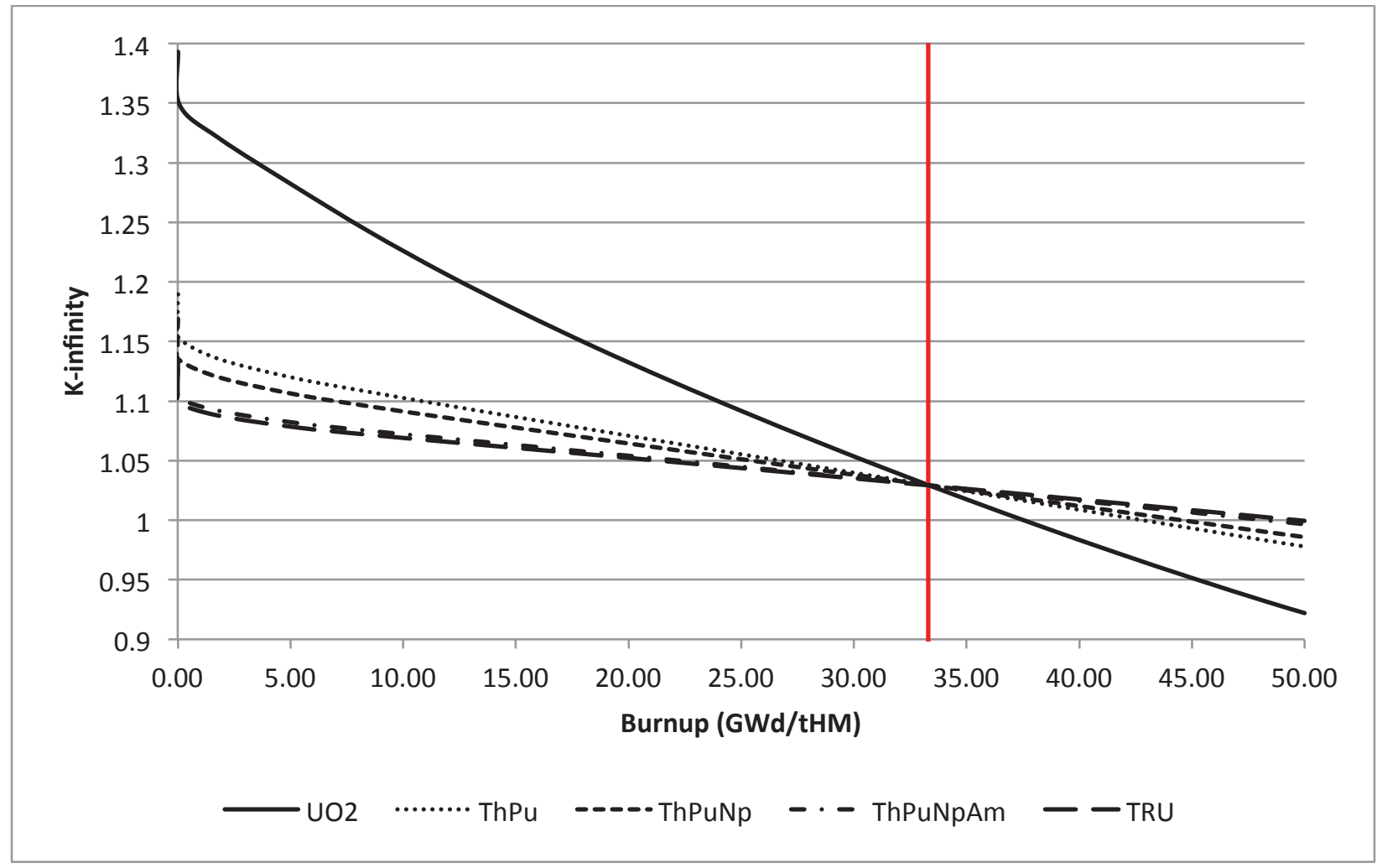

Figure 2.2 - K-infinity trends vs. burnup showing the intersection point at $33.33 \mathrm{GWd} / \mathrm{tHM}$.

The previous explained strategy ensures the different fuels will release the same amount of energy and thus allows relevant comparisons among them.

\subsection{DENSITY CALCULATION.}

The mixture densities have been calculated through the individual values of the single mixture $\left(\rho_{i}\right)$ and relative volume $\left(w_{i}\right)$ :

\footnotetext{
${ }^{1}$ Pu, PuNp, PuNpAm or TRU
} 


$$
\rho_{\text {mixture }}=\sum_{i=1}^{\# \text { mixtures }} \rho_{i} \times w_{i}
$$

The computed value has been corrected considering the fabrication packing factor $(f)[10]$ :

$$
\rho_{\text {mixture_final }}=\rho_{\text {mixture }} \times f
$$

The following individual densities have been considered:

- ThOx: $10.0 \mathrm{~g} / \mathrm{cc}$;

- TRUOx: $11.5 \mathrm{~g} / \mathrm{cc}$.

Considering as an example the Th-PuOx case, the previous relations become:

$$
\begin{aligned}
\rho_{\text {ThPuOX }} & =\left(\rho_{\text {ThOx }} \times w_{\text {Thox }}+\rho_{\text {PuOx }} \times w_{\text {PuOx }}\right) \times f \\
& =(10.0 \times 0.8488+11.5 \times 0.1512) \times 0.95=\mathbf{9 . 7 1 5} \mathbf{g} / \mathbf{c c}
\end{aligned}
$$




\section{RESULTS}

\subsection{SCALE6.1}

The table and figure below show the calculated $P u / P u N p / P u N p A m / T R U$ enrichment and $\mathrm{Pu}, \mathrm{Np}$, Am, other-TRU contents in the fuel assemblies necessary to maintain the same average burn-up at discharge of $50 \mathrm{GWd} / \mathrm{tHM}$.

\begin{tabular}{|c|c|c|c|c|c|}
\hline Case & $\begin{array}{c}\text { TRU } \\
\text { enrichment } \\
\text { (w/o\%) }\end{array}$ & $\begin{array}{c}\text { Pu } \\
\text { content } \\
\text { (w/o\%) }\end{array}$ & $\begin{array}{c}\text { Np } \\
\text { content } \\
(\mathbf{w} / \mathbf{o} \%)\end{array}$ & $\begin{array}{c}\text { Am } \\
\text { content } \\
(\mathbf{w} / \mathbf{0} \%)\end{array}$ & $\begin{array}{c}\text { Other-TRU } \\
\text { content } \\
\text { (w/o\%) }\end{array}$ \\
\hline$T h-P u$ & $11.05 \%$ & $11.05 \%$ & $0.000 \%$ & $0.000 \%$ & $0.000 \%$ \\
\hline$T h-P u N p$ & $13.70 \%$ & $12.98 \%$ & $0.72 \%$ & $0.000 \%$ & $0.000 \%$ \\
\hline$T h-P u N p A m$ & $17.55 \%$ & $15.81 \%$ & $0.88 \%$ & $0.87 \%$ & $0.000 \%$ \\
\hline$T h-T R U$ & $17.25 \%$ & $15.45 \%$ & $0.86 \%$ & $0.85 \%$ & $0.10 \%$ \\
\hline
\end{tabular}

Table 3.1 - TRU enrichment and main actinide contents..

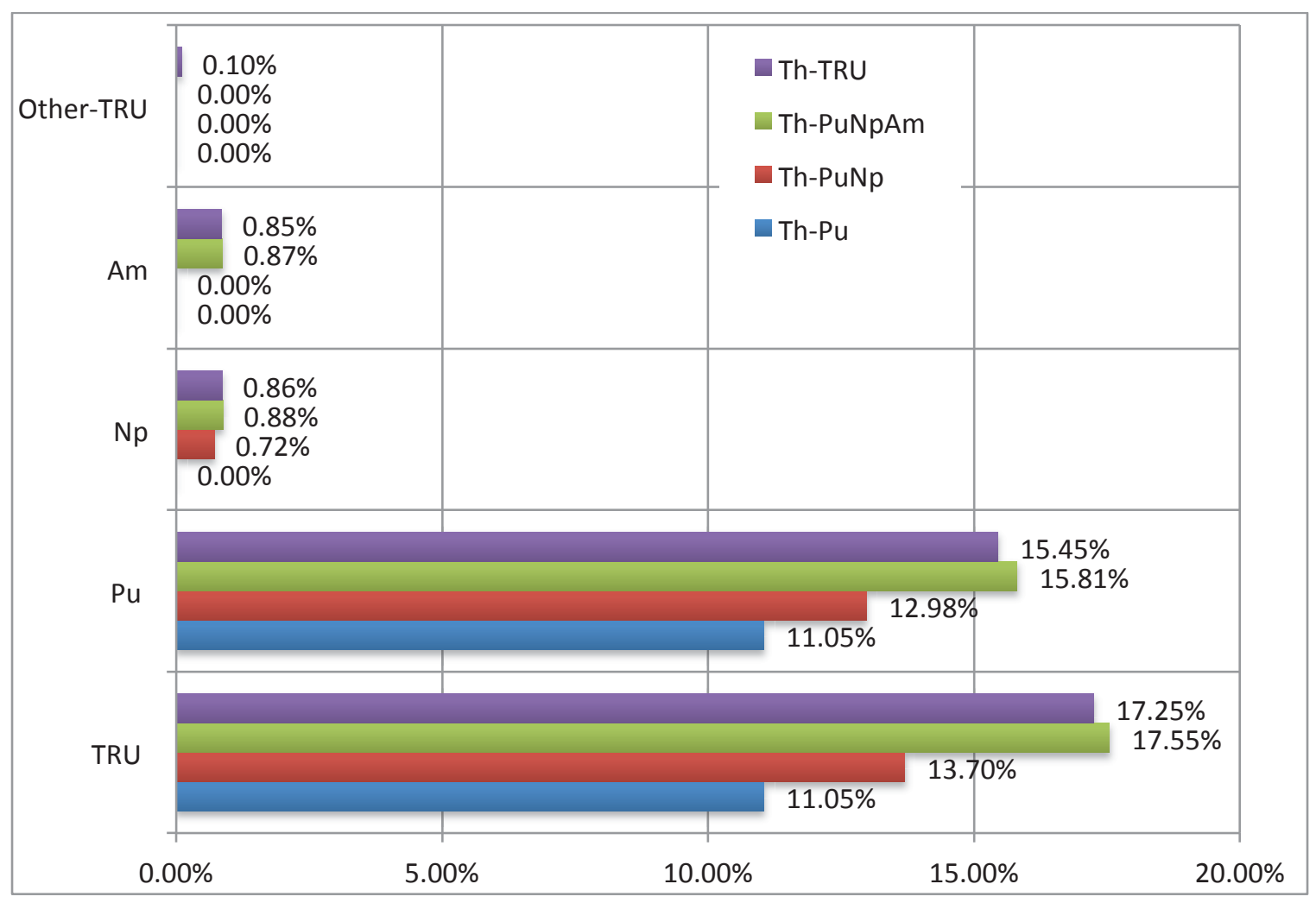

Figure 3.1 - TRU content in PWR Th-TRUOx fuel assemblies calculated with SCALE6.1.

The figure below shows the k-inf trend for each case and each cycle step (initial fuel aging, burning) against the $50 \mathrm{GWd} / \mathrm{tHM} \mathrm{UOX}(4.2 \%) \mathrm{SNF}$ reference case. 


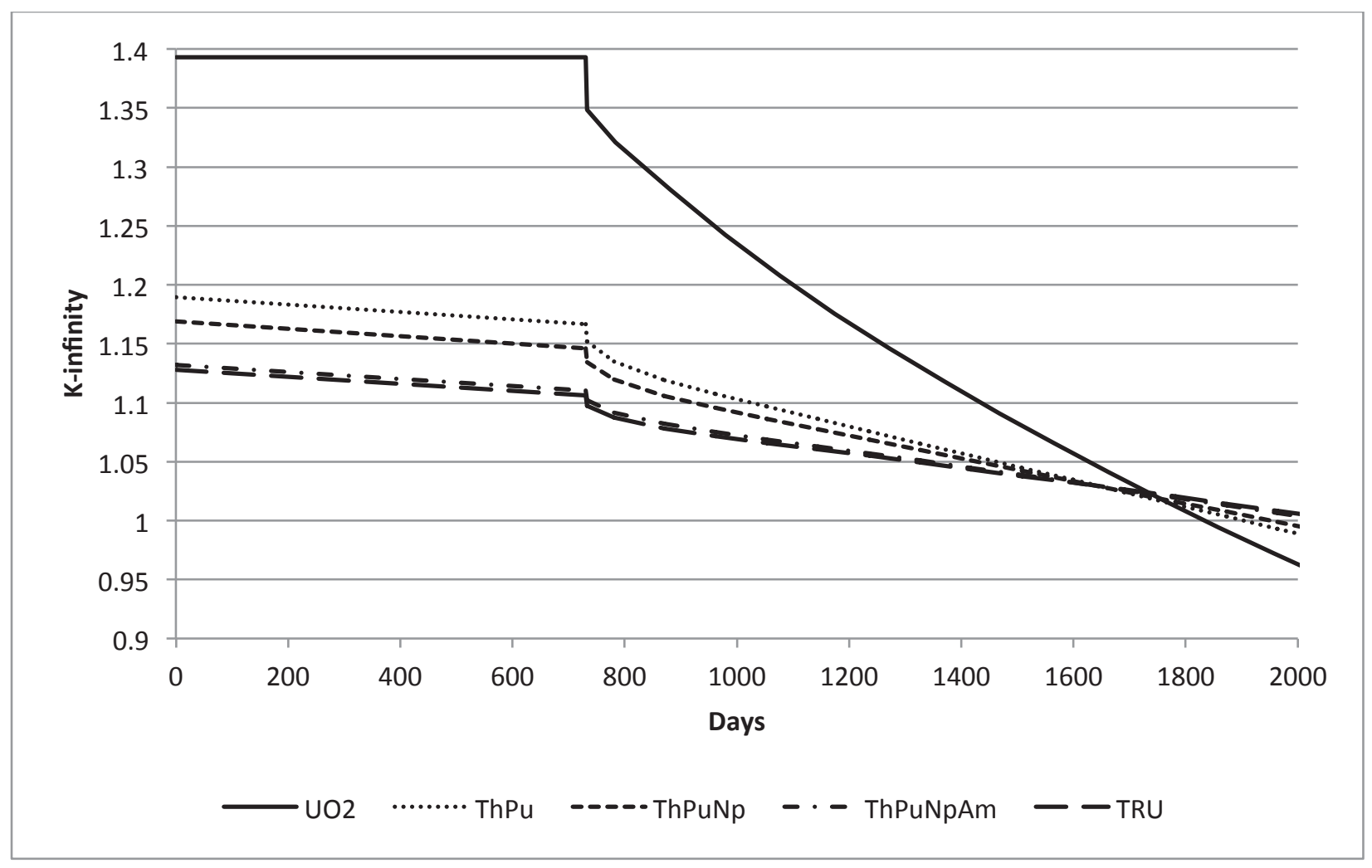

Figure 3.2 - $k$-infinitive trends.

The following table summarizes the isotopic input, after 2 years aging, and output compositions, at the average discharged burn-up of $50 \mathrm{GWd} / \mathrm{tHM}$, for the four cases taken in exam.

\begin{tabular}{|c|c|c|c|c|c|c|c|c|}
\hline \multirow{4}{*}{$\begin{array}{c}\text { Isotope } \\
\text { IDs }\end{array}$} & \multicolumn{8}{|c|}{ Isotopic input ( 2 yrs aging) and output mass vectors } \\
\hline & \multirow{2}{*}{\multicolumn{2}{|c|}{$\begin{array}{c}\text { ThPu } \\
k g / t I H M\end{array}$}} & \multirow{2}{*}{\multicolumn{2}{|c|}{$\begin{array}{l}\text { ThPuNp } \\
\mathrm{kg} / \mathrm{tIHM}\end{array}$}} & \multirow{2}{*}{\multicolumn{2}{|c|}{$\begin{array}{c}\text { ThPuNpAm } \\
\text { kg/tIHM }\end{array}$}} & \multirow{2}{*}{\multicolumn{2}{|c|}{$\begin{array}{l}\text { ThTRU } \\
\mathrm{kg} / \mathrm{tIHM}\end{array}$}} \\
\hline & & & & & & & & \\
\hline & Input & Output & Input & Output & Input & Output & Input & Output \\
\hline $\operatorname{Th} 228$ & $7.61 \mathrm{E}-09$ & $5.17 \mathrm{E}-04$ & $7.38 \mathrm{E}-09$ & $4.58 \mathrm{E}-04$ & $7.05 \mathrm{E}-09$ & $3.94 \mathrm{E}-04$ & $7.08 \mathrm{E}-09$ & $4.02 \mathrm{E}-04$ \\
\hline $\operatorname{Th} 229$ & $1.52 \mathrm{E}-14$ & $1.96 \mathrm{E}-04$ & $1.89 \mathrm{E}-11$ & $1.77 \mathrm{E}-04$ & $2.30 \mathrm{E}-11$ & $1.58 \mathrm{E}-04$ & $2.25 \mathrm{E}-11$ & $1.60 \mathrm{E}-04$ \\
\hline$T h 230$ & $1.28 \mathrm{E}-07$ & $1.56 \mathrm{E}-03$ & $1.50 \mathrm{E}-07$ & $1.58 \mathrm{E}-03$ & $1.83 \mathrm{E}-07$ & $1.62 \mathrm{E}-03$ & $1.79 \mathrm{E}-07$ & $1.63 \mathrm{E}-03$ \\
\hline Th232 & $8.89 \mathrm{E}+02$ & $8.65 \mathrm{E}+02$ & $8.63 \mathrm{E}+02$ & $8.40 \mathrm{E}+02$ & $8.24 \mathrm{E}+02$ & $8.03 \mathrm{E}+02$ & $8.27 \mathrm{E}+02$ & $8.06 \mathrm{E}+02$ \\
\hline$P a 231$ & $1.05 \mathrm{E}-11$ & $1.57 \mathrm{E}-01$ & $1.10 \mathrm{E}-11$ & $1.63 \mathrm{E}-01$ & $1.17 \mathrm{E}-11$ & $1.69 \mathrm{E}-01$ & $1.17 \mathrm{E}-11$ & $1.69 \mathrm{E}-01$ \\
\hline$U 232$ & $4.25 \mathrm{E}-10$ & $6.49 \mathrm{E}-02$ & $4.25 \mathrm{E}-10$ & $5.68 \mathrm{E}-02$ & $4.25 \mathrm{E}-10$ & $4.79 \mathrm{E}-02$ & $4.25 \mathrm{E}-10$ & $4.89 \mathrm{E}-02$ \\
\hline$U 233$ & $1.98 \mathrm{E}-09$ & $1.42 \mathrm{E}+01$ & $4.57 \mathrm{E}-06$ & $1.40 \mathrm{E}+01$ & $5.58 \mathrm{E}-06$ & $1.36 \mathrm{E}+01$ & $5.45 \mathrm{E}-06$ & $1.37 \mathrm{E}+01$ \\
\hline$U 234$ & $4.59 \mathrm{E}-02$ & $1.31 \mathrm{E}+00$ & $5.39 \mathrm{E}-02$ & $1.22 \mathrm{E}+00$ & $6.57 \mathrm{E}-02$ & $1.11 \mathrm{E}+00$ & $6.42 \mathrm{E}-02$ & $1.13 \mathrm{E}+00$ \\
\hline$U 235$ & $3.29 \mathrm{E}-03$ & $1.18 \mathrm{E}-01$ & $3.86 \mathrm{E}-03$ & $1.09 \mathrm{E}-01$ & $4.71 \mathrm{E}-03$ & $9.75 \mathrm{E}-02$ & $4.60 \mathrm{E}-03$ & $9.85 \mathrm{E}-02$ \\
\hline$U 236$ & $5.77 \mathrm{E}-03$ & $1.98 \mathrm{E}-02$ & $6.77 \mathrm{E}-03$ & $2.15 \mathrm{E}-02$ & $8.25 \mathrm{E}-03$ & $2.45 \mathrm{E}-02$ & $8.07 \mathrm{E}-03$ & $2.41 \mathrm{E}-02$ \\
\hline
\end{tabular}




\begin{tabular}{|c|c|c|c|c|c|c|c|c|}
\hline$U 238$ & 2.99E-05 & $9.60 \mathrm{E}-05$ & $3.51 \mathrm{E}-05$ & $1.07 \mathrm{E}-04$ & $4.28 \mathrm{E}-05$ & $1.27 \mathrm{E}-04$ & $4.18 \mathrm{E}-05$ & $1.25 \mathrm{E}-04$ \\
\hline$N p 237$ & $1.99 \mathrm{E}-03$ & $9.82 \mathrm{E}-03$ & $7.20 \mathrm{E}+00$ & $3.90 \mathrm{E}+00$ & $8.79 \mathrm{E}+00$ & $5.18 \mathrm{E}+00$ & $8.58 \mathrm{E}+00$ & $5.03 E+00$ \\
\hline Pu238 & $2.93 \mathrm{E}+00$ & $2.92 \mathrm{E}+00$ & $3.45 \mathrm{E}+00$ & $6.10 \mathrm{E}+00$ & $4.20 \mathrm{E}+00$ & $8.98 \mathrm{E}+00$ & $4.10 \mathrm{E}+00$ & $8.82 \mathrm{E}+00$ \\
\hline Pu239 & $5.82 \mathrm{E}+01$ & $1.52 \mathrm{E}+01$ & $6.84 \mathrm{E}+01$ & $2.40 \mathrm{E}+01$ & $8.33 \mathrm{E}+01$ & $3.78 \mathrm{E}+01$ & $8.14 \mathrm{E}+01$ & $3.63 \mathrm{E}+01$ \\
\hline Pu240 & $2.78 \mathrm{E}+01$ & $2.14 \mathrm{E}+01$ & $3.26 \mathrm{E}+01$ & $2.71 \mathrm{E}+01$ & $3.97 \mathrm{E}+01$ & $3.53 \mathrm{E}+01$ & $3.89 \mathrm{E}+01$ & $3.44 \mathrm{E}+01$ \\
\hline Pu241 & $1.20 \mathrm{E}+01$ & $1.25 \mathrm{E}+01$ & $1.41 \mathrm{E}+01$ & $1.48 \mathrm{E}+01$ & $1.72 \mathrm{E}+01$ & $1.75 \mathrm{E}+01$ & $1.68 \mathrm{E}+01$ & $1.72 \mathrm{E}+01$ \\
\hline Pu242 & $8.23 \mathrm{E}+00$ & $9.37 \mathrm{E}+00$ & $9.67 \mathrm{E}+00$ & $1.05 \mathrm{E}+01$ & $1.18 \mathrm{E}+01$ & $1.27 \mathrm{E}+01$ & $1.15 \mathrm{E}+01$ & $1.24 \mathrm{E}+01$ \\
\hline$A m 241$ & $1.22 \mathrm{E}+00$ & $1.57 \mathrm{E}+00$ & $1.44 \mathrm{E}+00$ & $2.06 \mathrm{E}+00$ & $7.72 \mathrm{E}+00$ & $5.25 \mathrm{E}+00$ & $7.55 \mathrm{E}+00$ & $5.07 \mathrm{E}+00$ \\
\hline$A m 242 m$ & $2.61 \mathrm{E}-08$ & $3.40 \mathrm{E}-02$ & $2.67 \mathrm{E}-08$ & $4.82 \mathrm{E}-02$ & $1.10 \mathrm{E}-02$ & $1.65 \mathrm{E}-01$ & $1.08 \mathrm{E}-02$ & $1.59 \mathrm{E}-01$ \\
\hline$A m 243$ & $1.44 \mathrm{E}-09$ & $2.30 \mathrm{E}+00$ & $1.45 \mathrm{E}-09$ & $2.43 \mathrm{E}+00$ & $2.65 \mathrm{E}+00$ & $3.81 \mathrm{E}+00$ & $2.59 \mathrm{E}+00$ & $3.75 \mathrm{E}+00$ \\
\hline$C m 242$ & $2.15 \mathrm{E}-11$ & $2.87 \mathrm{E}-01$ & $2.19 \mathrm{E}-11$ & $3.00 \mathrm{E}-01$ & $2.75 \mathrm{E}-05$ & $6.35 \mathrm{E}-01$ & $3.71 \mathrm{E}-05$ & $6.27 \mathrm{E}-01$ \\
\hline$C m 243$ & $4.51 \mathrm{E}-16$ & $1.32 \mathrm{E}-02$ & $4.51 \mathrm{E}-16$ & $1.38 \mathrm{E}-02$ & $4.56 \mathrm{E}-13$ & $3.62 \mathrm{E}-02$ & $8.46 \mathrm{E}-03$ & $3.81 \mathrm{E}-02$ \\
\hline $\mathrm{Cm} 244$ & $4.41 \mathrm{E}-16$ & $1.19 \mathrm{E}+00$ & $4.40 \mathrm{E}-16$ & $1.13 \mathrm{E}+00$ & 1.64E-09 & $2.15 \mathrm{E}+00$ & $8.54 \mathrm{E}-01$ & $2.65 \mathrm{E}+00$ \\
\hline$C m 245$ & $4.07 \mathrm{E}-16$ & 1.64E-01 & $5.96 \mathrm{E}-16$ & $1.52 \mathrm{E}-01$ & $6.25 \mathrm{E}-16$ & $3.14 \mathrm{E}-01$ & $7.38 \mathrm{E}-02$ & $4.71 \mathrm{E}-01$ \\
\hline $\mathrm{Cm} 246$ & $1.14 \mathrm{E}-09$ & $1.06 \mathrm{E}-02$ & $1.14 \mathrm{E}-09$ & $7.90 \mathrm{E}-03$ & $1.14 \mathrm{E}-09$ & $1.61 \mathrm{E}-02$ & $9.43 \mathrm{E}-03$ & 4.07E-02 \\
\hline$C m 247$ & $1.70 \mathrm{E}-17$ & $1.90 \mathrm{E}-04$ & $1.62 \mathrm{E}-17$ & $1.35 \mathrm{E}-04$ & $1.53 \mathrm{E}-17$ & $3.09 \mathrm{E}-04$ & $1.14 \mathrm{E}-04$ & $1.55 \mathrm{E}-03$ \\
\hline$C m 248$ & $3.58 \mathrm{E}-25$ & $9.21 \mathrm{E}-06$ & $3.03 \mathrm{E}-25$ & $5.65 \mathrm{E}-06$ & $2.42 \mathrm{E}-25$ & $1.25 \mathrm{E}-05$ & $1.58 \mathrm{E}-09$ & $1.77 \mathrm{E}-04$ \\
\hline$B k 249$ & $3.88 \mathrm{E}-36$ & $1.79 \mathrm{E}-07$ & $3.13 \mathrm{E}-36$ & $1.11 \mathrm{E}-07$ & $2.38 \mathrm{E}-36$ & $2.58 \mathrm{E}-07$ & $4.91 \mathrm{E}-10$ & $5.12 \mathrm{E}-06$ \\
\hline$C f 249$ & $0.00 \mathrm{E}+00$ & $5.14 \mathrm{E}-08$ & $0.00 \mathrm{E}+00$ & $3.34 \mathrm{E}-08$ & $0.00 \mathrm{E}+00$ & $9.11 \mathrm{E}-08$ & $2.91 \mathrm{E}-09$ & $2.99 \mathrm{E}-06$ \\
\hline \multicolumn{9}{|c|}{ Element input and output summary } \\
\hline IDs & $\%$ & $\%$ & $\%$ & $\%$ & $\%$ & $\%$ & $\%$ & $\%$ \\
\hline$T h$ & $88.94 \%$ & $86.52 \%$ & $86.29 \%$ & $84.03 \%$ & $82.44 \%$ & $80.34 \%$ & $82.74 \%$ & $80.62 \%$ \\
\hline$P a$ & $0.00 \%$ & $0.02 \%$ & $0.00 \%$ & $0.02 \%$ & $0.00 \%$ & $0.02 \%$ & $0.00 \%$ & $0.02 \%$ \\
\hline$U$ & $0.01 \%$ & $1.57 \%$ & $0.01 \%$ & $1.54 \%$ & $0.01 \%$ & $1.49 \%$ & $0.01 \%$ & $1.50 \%$ \\
\hline$N p$ & $0.00 \%$ & $0.00 \%$ & $0.72 \%$ & $0.39 \%$ & $0.88 \%$ & $0.52 \%$ & $0.86 \%$ & $0.50 \%$ \\
\hline$P u$ & $10.92 \%$ & $6.14 \%$ & $12.83 \%$ & $8.24 \%$ & $15.62 \%$ & $11.23 \%$ & $15.27 \%$ & $10.91 \%$ \\
\hline$A m$ & $0.12 \%$ & $0.39 \%$ & $0.14 \%$ & $0.45 \%$ & $1.04 \%$ & $0.92 \%$ & $1.02 \%$ & $0.90 \%$ \\
\hline $\mathrm{Cm}$ & $0.00 \%$ & $0.17 \%$ & $0.00 \%$ & $0.16 \%$ & $0.00 \%$ & $0.31 \%$ & $0.09 \%$ & $0.38 \%$ \\
\hline$B k$ & $0.00 \%$ & $0.00 \%$ & $0.00 \%$ & $0.00 \%$ & $0.00 \%$ & $0.00 \%$ & $0.00 \%$ & $0.00 \%$ \\
\hline$C f$ & $0.00 \%$ & $0.00 \%$ & $0.00 \%$ & $0.00 \%$ & $0.00 \%$ & $0.00 \%$ & $0.00 \%$ & $0.00 \%$ \\
\hline$F P$ & $0.00 \%$ & $5.09 \%$ & $0.00 \%$ & $5.09 \%$ & $0.00 \%$ & $5.08 \%$ & $0.00 \%$ & $5.08 \%$ \\
\hline
\end{tabular}

Table 3.2 - Mass Fraction input and output (summary). 


\section{CONCLUSIONS.}

This report presents the results of a neutronics analysis in which the option of a thorium-based fuel cycle has been shown.

The main objective of these calculations is quantify the TRU enrichment needed to operate a Thorium based reactor (PWR) in order to maintain the same average burnup at discharge (50 $\mathrm{GWd} / \mathrm{tHM}$ ) and, consequently, collect the data needed for further analysis.

It is important to recognize that these results are based on infinite lattice assembly calculations, assuming standard UOX fuel assembly parameters with homogeneously loaded fuel. While this approach increases the ability to retrofit thorium-based fuels into existing commercial PWRs with minimal or no changes required to reactor hardware, it does not represent the optimum performance achievable. Additionally more detailed studies (i.e. optimization of assembly and reactor parameters, calculation of void coefficients, etc.) would be required. 


\section{REFERENCES.}

1. A. Alfonsi, S. Bays, C.Rabiti, S. Piet, "Multi-Reactor Transmutation Analysis Utility (MRTAU,al): Verification", ," INL/EXT-11-21384 (2011).

2. S. Bays, S. Piet, M. Pope, G. Youinou, A. Dumontier, D. Hawn, "Transmutation Dynamics: Impacts of Multi-Recycling on Fuel Cycle Performances", INL/EXT-09-16857 (2009).

3. G. Youinou, S. Bays, "A Neutronic Analysis of TRU Recycling in PWRs Loaded with MOXUE Fuel (MOX with U-235 Enriched U Support)", INL/EXT-09-16091 (2009).

4. M. DeHart, "Lattice Physics Capabilities of the SCALE Code System Using TRITON", Physor Conference (2006).

5. S. Piet, N.Soelberg, et al., "Analyzing Losses: Transuranics Into Waste and Fission Products Into Recycled Fuel", INL/CON-10-20136 (2010).

6. A. Alfonsi, C. Rabiti, A. Epiney, Y. Wang, J. Cogliati, "PHISICS TOOLKIT: Multi-Reactor Transmutation Analysis Utility MRTAU”, Physor Conference (2012)

7. S. J. Piet, N. R. Soelberg, et al., "The FIT Model - Fuel Cycle Integration and Tradeoffs", INL/EXT-10-20190 (2011).

8. L.Gauld, O. Herman, et al., "Origen-S: Scale system module to calculate fuel depletion, Actinide transmutation, fission product buildup and decay, and associated radiation source terms", Oak Ridge National Laboratory (2009).

9. A.Alfonsi,G. Youinou, "Impact Of Fission Products Impurity On The Plutonium Content in PWR MOX Fuels", INL/EXT-12-26114, (2012.

10. FCO2012, “Assumptions for Fuel Cycle Analyses, Revision 1," Fuel Cycle Options - System Analysis and Integration, FCRD-FCO-2012-000026, April 10, 2012. 


\section{Appendix 1: Detailed isotopic mass fraction input (after 2-yrs fuel aging).}

\begin{tabular}{|c|c|c|c|c|}
\hline \multicolumn{5}{|c|}{ Isotopic input mass vector } \\
\hline Isotope & ThPu & ThPuNp & ThPuNpAm & ThTRU \\
\hline IDs & $\mathrm{kg} / \mathrm{tIH} M$ & $\mathrm{~kg} / \mathrm{tIHM}$ & $\mathrm{kg} / \mathrm{tIHM}$ & $\mathrm{kg} / \mathrm{tIHM}$ \\
\hline $\mathrm{He} 4$ & $9.73 \mathrm{E}-04$ & $1.14 \mathrm{E}-03$ & $1.72 \mathrm{E}-03$ & $2.80 \mathrm{E}-03$ \\
\hline$R a 228$ & $7.66 \mathrm{E}-08$ & $7.43 \mathrm{E}-08$ & $7.10 \mathrm{E}-08$ & $7.13 \mathrm{E}-08$ \\
\hline Th228 & 7.61E-09 & $7.38 \mathrm{E}-09$ & $7.05 \mathrm{E}-09$ & $7.08 \mathrm{E}-09$ \\
\hline Th229 & $1.52 \mathrm{E}-14$ & $1.89 \mathrm{E}-11$ & $2.30 \mathrm{E}-11$ & $2.25 \mathrm{E}-11$ \\
\hline Th230 & $1.28 \mathrm{E}-07$ & $1.50 \mathrm{E}-07$ & $1.83 \mathrm{E}-07$ & $1.79 \mathrm{E}-07$ \\
\hline Th231 & $3.81 \mathrm{E}-08$ & $3.77 \mathrm{E}-08$ & $3.73 \mathrm{E}-08$ & $3.75 \mathrm{E}-08$ \\
\hline Th232 & $8.89 \mathrm{E}+02$ & $8.63 \mathrm{E}+02$ & $8.24 \mathrm{E}+02$ & $8.27 \mathrm{E}+02$ \\
\hline Pa231 & $1.05 \mathrm{E}-11$ & $1.10 \mathrm{E}-11$ & $1.17 \mathrm{E}-11$ & $1.17 \mathrm{E}-11$ \\
\hline Pa233 & $1.87 \mathrm{E}-08$ & $2.62 \mathrm{E}-07$ & $3.14 \mathrm{E}-07$ & $3.08 \mathrm{E}-07$ \\
\hline Pa234 & 4.87E-16 & $1.70 \mathrm{E}-14$ & $1.90 \mathrm{E}-14$ & $1.88 \mathrm{E}-14$ \\
\hline U232 & $4.25 \mathrm{E}-10$ & $4.25 \mathrm{E}-10$ & $4.25 \mathrm{E}-10$ & $4.25 \mathrm{E}-10$ \\
\hline U233 & $1.98 \mathrm{E}-09$ & $4.57 \mathrm{E}-06$ & $5.58 \mathrm{E}-06$ & $5.45 \mathrm{E}-06$ \\
\hline$U 234$ & $4.59 \mathrm{E}-02$ & $5.40 \mathrm{E}-02$ & $6.57 \mathrm{E}-02$ & $6.42 \mathrm{E}-02$ \\
\hline$U 235$ & $3.29 \mathrm{E}-03$ & $3.86 \mathrm{E}-03$ & $4.71 \mathrm{E}-03$ & $4.60 \mathrm{E}-03$ \\
\hline$U 236$ & $5.77 \mathrm{E}-03$ & $6.77 \mathrm{E}-03$ & $8.25 \mathrm{E}-03$ & 8.07E-03 \\
\hline$U 237$ & $3.76 \mathrm{E}-07$ & $4.41 \mathrm{E}-07$ & $5.38 \mathrm{E}-07$ & $5.25 \mathrm{E}-07$ \\
\hline$U 238$ & $2.99 \mathrm{E}-05$ & $3.51 \mathrm{E}-05$ & $4.28 \mathrm{E}-05$ & $4.18 \mathrm{E}-05$ \\
\hline$N p 236$ & $3.46 \mathrm{E}-15$ & $1.28 \mathrm{E}-11$ & $1.62 \mathrm{E}-11$ & $1.59 \mathrm{E}-11$ \\
\hline$N p 237$ & $1.99 \mathrm{E}-03$ & $7.20 \mathrm{E}+00$ & $8.79 \mathrm{E}+00$ & $8.59 \mathrm{E}+00$ \\
\hline$N p 238$ & $2.14 \mathrm{E}-10$ & $6.78 \mathrm{E}-07$ & $7.20 \mathrm{E}-07$ & $7.14 \mathrm{E}-07$ \\
\hline$N p 239$ & $2.56 \mathrm{E}-13$ & $3.20 \mathrm{E}-13$ & 2.29E-06 & $2.23 \mathrm{E}-06$ \\
\hline Pu236 & $7.01 \mathrm{E}-10$ & $7.01 \mathrm{E}-10$ & $7.01 \mathrm{E}-10$ & $7.01 \mathrm{E}-10$ \\
\hline Pu237 & $3.52 \mathrm{E}-11$ & $4.22 \mathrm{E}-11$ & 5.32E-11 & $5.22 \mathrm{E}-11$ \\
\hline Pu238 & $2.93 \mathrm{E}+00$ & $3.45 \mathrm{E}+00$ & $4.20 \mathrm{E}+00$ & $4.10 \mathrm{E}+00$ \\
\hline Pu239 & $5.82 \mathrm{E}+01$ & $6.84 \mathrm{E}+01$ & $8.33 \mathrm{E}+01$ & $8.14 \mathrm{E}+01$ \\
\hline Pu240 & $2.78 \mathrm{E}+01$ & $3.26 \mathrm{E}+01$ & $3.97 \mathrm{E}+01$ & $3.89 \mathrm{E}+01$ \\
\hline Pu241 & $1.20 \mathrm{E}+01$ & $1.41 \mathrm{E}+01$ & $1.72 \mathrm{E}+01$ & $1.68 \mathrm{E}+01$ \\
\hline Pu242 & $8.23 \mathrm{E}+00$ & $9.67 \mathrm{E}+00$ & $1.18 \mathrm{E}+01$ & $1.15 \mathrm{E}+01$ \\
\hline Pu243 & $5.93 \mathrm{E}-07$ & $6.12 \mathrm{E}-07$ & $6.28 \mathrm{E}-07$ & $6.27 \mathrm{E}-07$ \\
\hline Pu244 & $2.27 \mathrm{E}-04$ & $3.41 \mathrm{E}-04$ & $3.41 \mathrm{E}-04$ & $3.41 \mathrm{E}-04$ \\
\hline Am241 & $1.22 \mathrm{E}+00$ & $1.44 \mathrm{E}+00$ & $7.72 \mathrm{E}+00$ & $7.55 \mathrm{E}+00$ \\
\hline Am242 & $2.16 \mathrm{E}-07$ & $2.20 \mathrm{E}-07$ & $1.07 \mathrm{E}-06$ & $1.07 \mathrm{E}-06$ \\
\hline Am242M & $2.61 \mathrm{E}-08$ & $2.67 \mathrm{E}-08$ & $1.10 \mathrm{E}-02$ & $1.08 \mathrm{E}-02$ \\
\hline Am243 & $1.44 \mathrm{E}-09$ & $1.45 \mathrm{E}-09$ & $2.65 \mathrm{E}+00$ & $2.59 \mathrm{E}+00$ \\
\hline $\mathrm{Cm} 242$ & $2.15 \mathrm{E}-11$ & $2.19 \mathrm{E}-11$ & $2.75 \mathrm{E}-05$ & $3.71 \mathrm{E}-05$ \\
\hline
\end{tabular}




\begin{tabular}{|c|c|c|c|c|}
\hline $\mathrm{Cm} 243$ & $4.51 \mathrm{E}-16$ & $4.51 \mathrm{E}-16$ & $4.56 \mathrm{E}-13$ & $8.46 \mathrm{E}-03$ \\
\hline $\mathrm{Cm} 244$ & $4.41 \mathrm{E}-16$ & $4.40 \mathrm{E}-16$ & 1.64E-09 & $8.54 \mathrm{E}-01$ \\
\hline $\mathrm{Cm} 245$ & $4.07 \mathrm{E}-16$ & $5.96 \mathrm{E}-16$ & $6.25 \mathrm{E}-16$ & $7.38 \mathrm{E}-02$ \\
\hline $\mathrm{Cm} 246$ & 1.14E-09 & $1.14 \mathrm{E}-09$ & $1.14 \mathrm{E}-09$ & $9.43 \mathrm{E}-03$ \\
\hline $\mathrm{Cm} 247$ & $1.70 \mathrm{E}-17$ & $1.62 \mathrm{E}-17$ & $1.53 \mathrm{E}-17$ & $1.14 \mathrm{E}-04$ \\
\hline $\mathrm{Cm} 248$ & $3.58 \mathrm{E}-25$ & $3.03 \mathrm{E}-25$ & $2.42 \mathrm{E}-25$ & $1.58 \mathrm{E}-09$ \\
\hline$B k 249$ & $3.88 \mathrm{E}-36$ & $3.13 \mathrm{E}-36$ & $2.38 \mathrm{E}-36$ & $4.91 \mathrm{E}-10$ \\
\hline$C f 249$ & $0.00 \mathrm{E}+00$ & $0.00 \mathrm{E}+00$ & $0.00 \mathrm{E}+00$ & $2.91 \mathrm{E}-09$ \\
\hline$Z n 70$ & $8.36 \mathrm{E}-14$ & $8.56 \mathrm{E}-14$ & 8.72E-14 & $8.72 \mathrm{E}-14$ \\
\hline Ga71 & $1.54 \mathrm{E}-14$ & $1.58 \mathrm{E}-14$ & $1.62 \mathrm{E}-14$ & $1.62 \mathrm{E}-14$ \\
\hline Ge72 & $8.32 \mathrm{E}-16$ & $8.38 \mathrm{E}-16$ & $8.46 \mathrm{E}-16$ & $8.47 \mathrm{E}-16$ \\
\hline Ge73 & $7.65 \mathrm{E}-15$ & $7.60 \mathrm{E}-15$ & $7.54 \mathrm{E}-15$ & $7.55 \mathrm{E}-15$ \\
\hline Ge74 & 4.17E-13 & $4.16 \mathrm{E}-13$ & $4.15 \mathrm{E}-13$ & $4.15 \mathrm{E}-13$ \\
\hline Ge76 & $2.15 \mathrm{E}-11$ & $2.15 \mathrm{E}-11$ & $2.16 \mathrm{E}-11$ & $2.16 \mathrm{E}-11$ \\
\hline As 75 & $1.13 \mathrm{E}-14$ & $1.13 \mathrm{E}-14$ & $1.12 \mathrm{E}-14$ & $1.12 \mathrm{E}-14$ \\
\hline $\mathrm{Se} 77$ & $2.52 \mathrm{E}-14$ & $2.53 \mathrm{E}-14$ & $2.54 \mathrm{E}-14$ & $2.54 \mathrm{E}-14$ \\
\hline $\mathrm{Se} 78$ & $3.56 \mathrm{E}-13$ & $3.52 \mathrm{E}-13$ & $3.46 \mathrm{E}-13$ & $3.46 \mathrm{E}-13$ \\
\hline Se79 & $4.87 \mathrm{E}-12$ & $4.80 \mathrm{E}-12$ & 4.70E-12 & $4.70 \mathrm{E}-12$ \\
\hline $\mathrm{Se} 80$ & $4.14 \mathrm{E}-10$ & $4.09 \mathrm{E}-10$ & $4.03 \mathrm{E}-10$ & $4.03 \mathrm{E}-10$ \\
\hline $\mathrm{Se} 82$ & $3.43 \mathrm{E}-09$ & $3.40 \mathrm{E}-09$ & $3.36 \mathrm{E}-09$ & 3.36E-09 \\
\hline$B r 79$ & $4.25 \mathrm{E}-15$ & $4.17 \mathrm{E}-15$ & $4.07 \mathrm{E}-15$ & $4.08 \mathrm{E}-15$ \\
\hline Br81 & $9.28 \mathrm{E}-12$ & $9.13 \mathrm{E}-12$ & 8.89E-12 & $8.90 \mathrm{E}-12$ \\
\hline$K r 82$ & $1.11 \mathrm{E}-13$ & $1.09 \mathrm{E}-13$ & $1.06 \mathrm{E}-13$ & $1.06 \mathrm{E}-13$ \\
\hline$K r 83$ & $3.92 \mathrm{E}-12$ & $3.87 \mathrm{E}-12$ & $3.78 \mathrm{E}-12$ & $3.78 \mathrm{E}-12$ \\
\hline$K r 84$ & $8.92 \mathrm{E}-11$ & $8.79 \mathrm{E}-11$ & $8.58 \mathrm{E}-11$ & $8.58 \mathrm{E}-11$ \\
\hline$K r 85$ & $2.62 \mathrm{E}-10$ & $2.59 \mathrm{E}-10$ & $2.56 \mathrm{E}-10$ & $2.55 \mathrm{E}-10$ \\
\hline $\mathrm{Kr} 86$ & $3.56 \mathrm{E}-09$ & 3.54E-09 & $3.51 \mathrm{E}-09$ & $3.51 \mathrm{E}-09$ \\
\hline$R b 85$ & $2.84 \mathrm{E}-12$ & $2.84 \mathrm{E}-12$ & $2.83 \mathrm{E}-12$ & $2.83 \mathrm{E}-12$ \\
\hline$R b 87$ & $3.72 \mathrm{E}-10$ & $3.66 \mathrm{E}-10$ & $3.56 \mathrm{E}-10$ & $3.56 \mathrm{E}-10$ \\
\hline Sr86 & $2.44 \mathrm{E}-14$ & $2.39 \mathrm{E}-14$ & 2.32E-14 & $2.33 \mathrm{E}-14$ \\
\hline Sr87 & $5.83 \mathrm{E}-13$ & $5.73 \mathrm{E}-13$ & $5.57 \mathrm{E}-13$ & $5.57 \mathrm{E}-13$ \\
\hline $\operatorname{Sr} 88$ & $3.11 \mathrm{E}-11$ & $3.07 \mathrm{E}-11$ & $3.01 \mathrm{E}-11$ & $3.01 \mathrm{E}-11$ \\
\hline Sr89 & $4.25 \mathrm{E}-10$ & $4.17 \mathrm{E}-10$ & $4.06 \mathrm{E}-10$ & $4.06 \mathrm{E}-10$ \\
\hline $\operatorname{Sr} 90$ & $3.27 \mathrm{E}-09$ & 3.22E-09 & $3.15 \mathrm{E}-09$ & $3.15 \mathrm{E}-09$ \\
\hline$Y 89$ & $6.64 \mathrm{E}-12$ & $6.62 \mathrm{E}-12$ & $6.57 \mathrm{E}-12$ & $6.57 \mathrm{E}-12$ \\
\hline$Y 90$ & $2.38 \mathrm{E}-12$ & $2.33 \mathrm{E}-12$ & $2.28 \mathrm{E}-12$ & $2.28 \mathrm{E}-12$ \\
\hline$Y 91$ & $3.28 \mathrm{E}-11$ & $3.24 \mathrm{E}-11$ & $3.18 \mathrm{E}-11$ & $3.18 \mathrm{E}-11$ \\
\hline $\mathrm{Zr} 90$ & $3.80 \mathrm{E}-13$ & $3.79 \mathrm{E}-13$ & $3.76 \mathrm{E}-13$ & $3.76 \mathrm{E}-13$ \\
\hline $\mathrm{Zr} 91$ & $9.56 \mathrm{E}-12$ & $9.54 \mathrm{E}-12$ & $9.48 \mathrm{E}-12$ & $9.48 \mathrm{E}-12$ \\
\hline $\mathrm{Zr} 92$ & $1.48 \mathrm{E}-11$ & $1.47 \mathrm{E}-11$ & $1.46 \mathrm{E}-11$ & $1.46 \mathrm{E}-11$ \\
\hline
\end{tabular}




\begin{tabular}{|c|c|c|c|c|}
\hline $\mathrm{Zr} 93$ & $5.89 \mathrm{E}-11$ & $5.82 \mathrm{E}-11$ & $5.71 \mathrm{E}-11$ & $5.71 \mathrm{E}-11$ \\
\hline $\mathrm{Zr} 94$ & $9.49 \mathrm{E}-10$ & $9.34 \mathrm{E}-10$ & $9.12 \mathrm{E}-10$ & $9.12 \mathrm{E}-10$ \\
\hline $\mathrm{Zr95}$ & $4.13 \mathrm{E}-09$ & $4.08 \mathrm{E}-09$ & 4.01E-09 & $4.01 \mathrm{E}-09$ \\
\hline Zr96 & $1.00 \mathrm{E}-07$ & $1.00 \mathrm{E}-07$ & $9.96 \mathrm{E}-08$ & $9.95 \mathrm{E}-08$ \\
\hline $\mathrm{Nb94}$ & $2.50 \mathrm{E}-13$ & $2.46 \mathrm{E}-13$ & $2.42 \mathrm{E}-13$ & $2.42 \mathrm{E}-13$ \\
\hline Nb95 & $2.71 \mathrm{E}-11$ & $2.67 \mathrm{E}-11$ & $2.61 \mathrm{E}-11$ & $2.74 \mathrm{E}-11$ \\
\hline Nb95M & 4.62E-12 & $4.54 \mathrm{E}-12$ & $4.43 \mathrm{E}-12$ & 4.44E-12 \\
\hline Mo95 & $1.89 \mathrm{E}-11$ & $1.88 \mathrm{E}-11$ & $1.87 \mathrm{E}-11$ & $1.87 \mathrm{E}-11$ \\
\hline Mo96 & $1.56 \mathrm{E}-13$ & $1.54 \mathrm{E}-13$ & $1.52 \mathrm{E}-13$ & $1.52 \mathrm{E}-13$ \\
\hline Mo97 & $2.83 \mathrm{E}-11$ & $2.82 \mathrm{E}-11$ & $2.79 \mathrm{E}-11$ & $2.79 \mathrm{E}-11$ \\
\hline Mo98 & $2.73 \mathrm{E}-08$ & $2.73 \mathrm{E}-08$ & $2.71 \mathrm{E}-08$ & $2.71 \mathrm{E}-08$ \\
\hline Mo100 & $1.47 \mathrm{E}-07$ & $1.47 \mathrm{E}-07$ & $1.46 \mathrm{E}-07$ & $1.46 \mathrm{E}-07$ \\
\hline Tc98 & $1.12 \mathrm{E}-14$ & $1.10 \mathrm{E}-14$ & $1.09 \mathrm{E}-14$ & $1.10 \mathrm{E}-14$ \\
\hline Tc99 & $2.95 \mathrm{E}-11$ & $2.94 \mathrm{E}-11$ & $2.93 \mathrm{E}-11$ & $2.93 \mathrm{E}-11$ \\
\hline Ru99 & $1.35 \mathrm{E}-15$ & $1.35 \mathrm{E}-15$ & $1.34 \mathrm{E}-15$ & $1.34 \mathrm{E}-15$ \\
\hline Ru100 & $2.82 \mathrm{E}-12$ & $2.77 \mathrm{E}-12$ & $2.74 \mathrm{E}-12$ & $2.74 \mathrm{E}-12$ \\
\hline Ru101 & $3.17 \mathrm{E}-11$ & $3.16 \mathrm{E}-11$ & $3.15 \mathrm{E}-11$ & $3.15 \mathrm{E}-11$ \\
\hline Ru102 & $1.57 \mathrm{E}-09$ & $1.56 \mathrm{E}-09$ & $1.54 \mathrm{E}-09$ & $1.54 \mathrm{E}-09$ \\
\hline Ru103 & $2.83 \mathrm{E}-09$ & $2.81 \mathrm{E}-09$ & 2.79E-09 & $2.79 \mathrm{E}-09$ \\
\hline Ru104 & $9.25 \mathrm{E}-10$ & $9.12 \mathrm{E}-10$ & $8.95 \mathrm{E}-10$ & $8.97 \mathrm{E}-10$ \\
\hline Ru106 & $2.87 \mathrm{E}-08$ & $2.86 \mathrm{E}-08$ & $2.85 \mathrm{E}-08$ & $2.86 \mathrm{E}-08$ \\
\hline$R h 103$ & $3.17 \mathrm{E}-11$ & $3.17 \mathrm{E}-11$ & $3.15 \mathrm{E}-11$ & $3.15 \mathrm{E}-11$ \\
\hline Rh103M & $9.62 \mathrm{E}-15$ & $9.52 \mathrm{E}-15$ & $9.49 \mathrm{E}-15$ & $9.58 \mathrm{E}-15$ \\
\hline Rh106 & $6.54 \mathrm{E}-12$ & $6.69 \mathrm{E}-12$ & $7.21 \mathrm{E}-12$ & $7.38 \mathrm{E}-12$ \\
\hline Pd104 & $8.13 \mathrm{E}-15$ & $8.03 \mathrm{E}-15$ & 7.99E-15 & $8.09 \mathrm{E}-15$ \\
\hline Pd105 & $2.55 \mathrm{E}-11$ & $2.55 \mathrm{E}-11$ & $2.54 \mathrm{E}-11$ & $2.54 \mathrm{E}-11$ \\
\hline$P d 106$ & $1.76 \mathrm{E}-11$ & $1.76 \mathrm{E}-11$ & $1.78 \mathrm{E}-11$ & $1.78 \mathrm{E}-11$ \\
\hline$P d 107$ & $3.30 \mathrm{E}-11$ & $3.27 \mathrm{E}-11$ & $3.24 \mathrm{E}-11$ & $3.24 \mathrm{E}-11$ \\
\hline$P d 108$ & $6.25 \mathrm{E}-10$ & $6.23 \mathrm{E}-10$ & $6.26 \mathrm{E}-10$ & $6.29 \mathrm{E}-10$ \\
\hline$P d 110$ & $9.10 \mathrm{E}-09$ & $9.14 \mathrm{E}-09$ & 9.30E-09 & $9.38 \mathrm{E}-09$ \\
\hline Ag109 & $8.14 \mathrm{E}-12$ & $8.14 \mathrm{E}-12$ & $8.18 \mathrm{E}-12$ & $8.20 \mathrm{E}-12$ \\
\hline Ag110M & $1.67 \mathrm{E}-14$ & $1.76 \mathrm{E}-14$ & $3.93 \mathrm{E}-14$ & 7.63E-14 \\
\hline $\operatorname{In} 115$ & $2.30 \mathrm{E}-13$ & $2.32 \mathrm{E}-13$ & $2.36 \mathrm{E}-13$ & $2.40 \mathrm{E}-13$ \\
\hline Sn 115 & $1.16 \mathrm{E}-14$ & $1.16 \mathrm{E}-14$ & $1.19 \mathrm{E}-14$ & $1.20 \mathrm{E}-14$ \\
\hline Sn116 & $1.81 \mathrm{E}-15$ & $1.93 \mathrm{E}-15$ & $2.12 \mathrm{E}-15$ & $2.10 \mathrm{E}-15$ \\
\hline Sn 117 & $2.27 \mathrm{E}-13$ & $2.28 \mathrm{E}-13$ & $2.30 \mathrm{E}-13$ & $2.30 \mathrm{E}-13$ \\
\hline Sn118 & $2.83 \mathrm{E}-12$ & $2.89 \mathrm{E}-12$ & $2.99 \mathrm{E}-12$ & $2.98 \mathrm{E}-12$ \\
\hline Sn119 & $2.46 \mathrm{E}-12$ & $2.47 \mathrm{E}-12$ & $2.51 \mathrm{E}-12$ & $2.51 \mathrm{E}-12$ \\
\hline Sn120 & $1.56 \mathrm{E}-10$ & $1.57 \mathrm{E}-10$ & $1.61 \mathrm{E}-10$ & $1.61 \mathrm{E}-10$ \\
\hline Sn121 & $1.69 \mathrm{E}-10$ & $1.70 \mathrm{E}-10$ & $1.72 \mathrm{E}-10$ & $1.72 \mathrm{E}-10$ \\
\hline
\end{tabular}




\begin{tabular}{|c|c|c|c|c|}
\hline Sn121M & $3.20 \mathrm{E}-11$ & $3.22 \mathrm{E}-11$ & $3.28 \mathrm{E}-11$ & $3.29 \mathrm{E}-11$ \\
\hline Sn 122 & $1.30 \mathrm{E}-09$ & $1.31 \mathrm{E}-09$ & 1.33E-09 & $1.33 \mathrm{E}-09$ \\
\hline Sn123 & $4.81 \mathrm{E}-10$ & $4.84 \mathrm{E}-10$ & $4.91 \mathrm{E}-10$ & $4.91 \mathrm{E}-10$ \\
\hline Sn 124 & $2.86 \mathrm{E}-09$ & $2.86 \mathrm{E}-09$ & $2.87 \mathrm{E}-09$ & $2.87 \mathrm{E}-09$ \\
\hline Sn 126 & 8.09E-09 & 8.08E-09 & 8.08E-09 & $8.08 \mathrm{E}-09$ \\
\hline$S b 121$ & $2.26 \mathrm{E}-13$ & $2.30 \mathrm{E}-13$ & $2.40 \mathrm{E}-13$ & $2.41 \mathrm{E}-13$ \\
\hline$S b 123$ & $1.83 \mathrm{E}-11$ & $1.82 \mathrm{E}-11$ & $1.82 \mathrm{E}-11$ & $1.82 \mathrm{E}-11$ \\
\hline $\mathrm{Sb} 124$ & $4.87 \mathrm{E}-12$ & $4.88 \mathrm{E}-12$ & $4.95 \mathrm{E}-12$ & $4.96 \mathrm{E}-12$ \\
\hline$S b 125$ & $3.94 \mathrm{E}-11$ & $3.94 \mathrm{E}-11$ & $3.97 \mathrm{E}-11$ & $3.97 \mathrm{E}-11$ \\
\hline$S b 126$ & 4.97E-11 & $4.96 \mathrm{E}-11$ & 4.97E-11 & $4.97 \mathrm{E}-11$ \\
\hline $\mathrm{Te} 122$ & $1.95 \mathrm{E}-15$ & $2.00 \mathrm{E}-15$ & $2.16 \mathrm{E}-15$ & $2.23 \mathrm{E}-15$ \\
\hline $\mathrm{Te} 124$ & $1.64 \mathrm{E}-13$ & $1.64 \mathrm{E}-13$ & $1.67 \mathrm{E}-13$ & $1.67 \mathrm{E}-13$ \\
\hline $\mathrm{Te} 125$ & $2.06 \mathrm{E}-13$ & $2.06 \mathrm{E}-13$ & $2.07 \mathrm{E}-13$ & $2.07 \mathrm{E}-13$ \\
\hline $\mathrm{Te} 125 \mathrm{M}$ & $7.01 \mathrm{E}-15$ & $8.19 \mathrm{E}-15$ & $1.02 \mathrm{E}-14$ & $9.78 \mathrm{E}-15$ \\
\hline $\mathrm{Te} 126$ & $4.72 \mathrm{E}-13$ & $4.80 \mathrm{E}-13$ & $4.93 \mathrm{E}-13$ & $4.91 \mathrm{E}-13$ \\
\hline $\mathrm{Te} 127$ & $6.52 \mathrm{E}-14$ & $7.59 \mathrm{E}-14$ & 8.72E-14 & $8.59 \mathrm{E}-14$ \\
\hline $\mathrm{Te} 127 \mathrm{M}$ & $1.46 \mathrm{E}-13$ & $1.97 \mathrm{E}-13$ & $2.51 \mathrm{E}-13$ & $2.45 \mathrm{E}-13$ \\
\hline $\mathrm{Te} 128$ & $2.87 \mathrm{E}-11$ & $2.87 \mathrm{E}-11$ & $2.85 \mathrm{E}-11$ & $2.85 \mathrm{E}-11$ \\
\hline Te129M & $2.05 \mathrm{E}-11$ & $2.11 \mathrm{E}-11$ & $2.19 \mathrm{E}-11$ & $2.26 \mathrm{E}-11$ \\
\hline Te130 & 7.93E-09 & 7.84E-09 & 7.70E-09 & 7.70E-09 \\
\hline$I 127$ & $2.40 \mathrm{E}-12$ & $2.39 \mathrm{E}-12$ & $2.37 \mathrm{E}-12$ & $2.37 \mathrm{E}-12$ \\
\hline$I 129$ & $6.89 \mathrm{E}-12$ & $6.88 \mathrm{E}-12$ & $6.85 \mathrm{E}-12$ & $6.86 \mathrm{E}-12$ \\
\hline $\mathrm{Xe} 128$ & $1.39 \mathrm{E}-14$ & $1.36 \mathrm{E}-14$ & $1.31 \mathrm{E}-14$ & $1.31 \mathrm{E}-14$ \\
\hline Xe130 & $2.47 \mathrm{E}-13$ & $2.43 \mathrm{E}-13$ & $2.37 \mathrm{E}-13$ & $2.37 \mathrm{E}-13$ \\
\hline Xe131 & $2.10 \mathrm{E}-11$ & $2.10 \mathrm{E}-11$ & $2.09 \mathrm{E}-11$ & $2.08 \mathrm{E}-11$ \\
\hline Xel32 & $9.07 \mathrm{E}-11$ & $8.98 \mathrm{E}-11$ & $8.86 \mathrm{E}-11$ & $8.87 \mathrm{E}-11$ \\
\hline Xe134 & 9.63E-09 & $9.52 \mathrm{E}-09$ & 9.39E-09 & $9.39 \mathrm{E}-09$ \\
\hline Xe136 & $1.62 \mathrm{E}-07$ & $1.60 \mathrm{E}-07$ & $1.58 \mathrm{E}-07$ & $1.58 \mathrm{E}-07$ \\
\hline Cs 133 & $4.02 \mathrm{E}-11$ & $4.02 \mathrm{E}-11$ & $4.00 \mathrm{E}-11$ & $4.00 \mathrm{E}-11$ \\
\hline Cs 134 & $1.27 \mathrm{E}-11$ & $1.26 \mathrm{E}-11$ & $1.25 \mathrm{E}-11$ & $1.25 \mathrm{E}-11$ \\
\hline Cs 135 & $2.86 \mathrm{E}-10$ & $2.83 \mathrm{E}-10$ & $2.80 \mathrm{E}-10$ & $2.81 \mathrm{E}-10$ \\
\hline Cs 136 & $1.94 \mathrm{E}-09$ & $1.93 \mathrm{E}-09$ & $1.93 \mathrm{E}-09$ & $1.94 \mathrm{E}-09$ \\
\hline Cs 137 & $3.24 \mathrm{E}-08$ & $3.22 \mathrm{E}-08$ & $3.20 \mathrm{E}-08$ & $3.21 \mathrm{E}-08$ \\
\hline Bal34 & $9.70 \mathrm{E}-15$ & $9.69 \mathrm{E}-15$ & $9.88 \mathrm{E}-15$ & $9.91 \mathrm{E}-15$ \\
\hline Ba135 & $2.30 \mathrm{E}-13$ & $2.27 \mathrm{E}-13$ & $2.25 \mathrm{E}-13$ & $2.26 \mathrm{E}-13$ \\
\hline Ba136 & $5.82 \mathrm{E}-10$ & $5.78 \mathrm{E}-10$ & $5.79 \mathrm{E}-10$ & $5.81 \mathrm{E}-10$ \\
\hline Ba137 & $7.95 \mathrm{E}-11$ & $7.83 \mathrm{E}-11$ & 7.70E-11 & 7.72E-11 \\
\hline Ba137M & $1.65 \mathrm{E}-10$ & $1.63 \mathrm{E}-10$ & $1.61 \mathrm{E}-10$ & $1.61 \mathrm{E}-10$ \\
\hline Ba138 & $2.32 \mathrm{E}-09$ & 2.29E-09 & 2.27E-09 & $2.27 \mathrm{E}-09$ \\
\hline Bal 40 & $7.30 \mathrm{E}-08$ & 7.22E-08 & 7.09E-08 & $7.10 \mathrm{E}-08$ \\
\hline
\end{tabular}




\begin{tabular}{|c|c|c|c|c|}
\hline$L a 138$ & $2.36 \mathrm{E}-12$ & $2.33 \mathrm{E}-12$ & $2.33 \mathrm{E}-12$ & $2.35 \mathrm{E}-12$ \\
\hline La139 & $8.86 \mathrm{E}-11$ & $8.79 \mathrm{E}-11$ & $8.76 \mathrm{E}-11$ & $8.78 \mathrm{E}-11$ \\
\hline La140 & $4.01 \mathrm{E}-10$ & $3.97 \mathrm{E}-10$ & $4.00 \mathrm{E}-10$ & $4.03 \mathrm{E}-10$ \\
\hline $\mathrm{Ce} 140$ & $3.36 \mathrm{E}-11$ & $3.36 \mathrm{E}-11$ & $3.35 \mathrm{E}-11$ & $3.35 \mathrm{E}-11$ \\
\hline $\mathrm{Ce} 141$ & $1.07 \mathrm{E}-11$ & $1.06 \mathrm{E}-11$ & $1.05 \mathrm{E}-11$ & $1.06 \mathrm{E}-11$ \\
\hline $\mathrm{Ce} 142$ & $1.91 \mathrm{E}-10$ & $1.89 \mathrm{E}-10$ & $1.88 \mathrm{E}-10$ & $1.89 \mathrm{E}-10$ \\
\hline $\mathrm{Ce} 144$ & $2.07 \mathrm{E}-08$ & $2.06 \mathrm{E}-08$ & $2.06 \mathrm{E}-08$ & $2.06 \mathrm{E}-08$ \\
\hline $\operatorname{Pr} 141$ & $3.20 \mathrm{E}-11$ & $3.20 \mathrm{E}-11$ & $3.19 \mathrm{E}-11$ & $3.19 \mathrm{E}-11$ \\
\hline $\operatorname{Pr} 143$ & $1.44 \mathrm{E}-12$ & $1.42 \mathrm{E}-12$ & $1.43 \mathrm{E}-12$ & $1.44 \mathrm{E}-12$ \\
\hline $\operatorname{Pr} 144$ & $1.72 \mathrm{E}-12$ & $1.71 \mathrm{E}-12$ & $1.73 \mathrm{E}-12$ & $1.74 \mathrm{E}-12$ \\
\hline$N d 142$ & $5.61 \mathrm{E}-16$ & $5.61 \mathrm{E}-16$ & $5.61 \mathrm{E}-16$ & $5.61 \mathrm{E}-16$ \\
\hline$N d 143$ & $2.83 \mathrm{E}-11$ & $2.83 \mathrm{E}-11$ & $2.82 \mathrm{E}-11$ & $2.82 \mathrm{E}-11$ \\
\hline$N d 144$ & $2.02 \mathrm{E}-11$ & $2.02 \mathrm{E}-11$ & $2.02 \mathrm{E}-11$ & $2.02 \mathrm{E}-11$ \\
\hline$N d 145$ & $1.98 \mathrm{E}-11$ & $1.98 \mathrm{E}-11$ & $1.98 \mathrm{E}-11$ & $1.98 \mathrm{E}-11$ \\
\hline$N d 146$ & $2.56 \mathrm{E}-11$ & $2.56 \mathrm{E}-11$ & $2.57 \mathrm{E}-11$ & $2.58 \mathrm{E}-11$ \\
\hline$N d 147$ & $1.70 \mathrm{E}-10$ & $1.69 \mathrm{E}-10$ & $1.68 \mathrm{E}-10$ & $1.68 \mathrm{E}-10$ \\
\hline$N d 148$ & $2.17 \mathrm{E}-09$ & $2.14 \mathrm{E}-09$ & $2.11 \mathrm{E}-09$ & $2.11 \mathrm{E}-09$ \\
\hline$N d 150$ & $3.15 \mathrm{E}-08$ & $3.15 \mathrm{E}-08$ & $3.15 \mathrm{E}-08$ & $3.15 \mathrm{E}-08$ \\
\hline$P m 147$ & $7.91 \mathrm{E}-12$ & $7.91 \mathrm{E}-12$ & $7.90 \mathrm{E}-12$ & $7.91 \mathrm{E}-12$ \\
\hline$P m 148$ & $6.01 \mathrm{E}-14$ & $6.13 \mathrm{E}-14$ & $6.61 \mathrm{E}-14$ & $6.77 \mathrm{E}-14$ \\
\hline$P m 148 M$ & $2.06 \mathrm{E}-13$ & $2.09 \mathrm{E}-13$ & $2.21 \mathrm{E}-13$ & $2.24 \mathrm{E}-13$ \\
\hline Sm147 & $5.33 \mathrm{E}-12$ & $5.33 \mathrm{E}-12$ & $5.33 \mathrm{E}-12$ & $5.33 \mathrm{E}-12$ \\
\hline $\operatorname{Sm} 148$ & $6.93 \mathrm{E}-16$ & $6.94 \mathrm{E}-16$ & $7.00 \mathrm{E}-16$ & $7.02 \mathrm{E}-16$ \\
\hline $\operatorname{Sm} 149$ & $8.22 \mathrm{E}-12$ & $8.23 \mathrm{E}-12$ & $8.24 \mathrm{E}-12$ & $8.24 \mathrm{E}-12$ \\
\hline $\operatorname{Sm} 150$ & $1.52 \mathrm{E}-13$ & $1.51 \mathrm{E}-13$ & $1.53 \mathrm{E}-13$ & $1.54 \mathrm{E}-13$ \\
\hline$S m 151$ & $6.71 \mathrm{E}-12$ & $6.71 \mathrm{E}-12$ & $6.73 \mathrm{E}-12$ & $6.74 \mathrm{E}-12$ \\
\hline $\operatorname{Sm} 152$ & $5.59 \mathrm{E}-11$ & $5.55 \mathrm{E}-11$ & $5.55 \mathrm{E}-11$ & $5.57 \mathrm{E}-11$ \\
\hline$S m 154$ & $7.23 \mathrm{E}-10$ & 7.19E-10 & $7.19 \mathrm{E}-10$ & $7.23 \mathrm{E}-10$ \\
\hline Eu151 & $7.81 \mathrm{E}-14$ & $7.82 \mathrm{E}-14$ & $7.83 \mathrm{E}-14$ & $7.84 \mathrm{E}-14$ \\
\hline Eu152 & $2.33 \mathrm{E}-15$ & $2.34 \mathrm{E}-15$ & $2.45 \mathrm{E}-15$ & $2.50 \mathrm{E}-15$ \\
\hline Eu153 & $2.76 \mathrm{E}-12$ & $2.77 \mathrm{E}-12$ & $2.80 \mathrm{E}-12$ & $2.81 \mathrm{E}-12$ \\
\hline Eu154 & $6.29 \mathrm{E}-13$ & $6.28 \mathrm{E}-13$ & $6.43 \mathrm{E}-13$ & $6.57 \mathrm{E}-13$ \\
\hline Eu155 & $1.83 \mathrm{E}-11$ & $1.83 \mathrm{E}-11$ & $1.87 \mathrm{E}-11$ & $1.89 \mathrm{E}-11$ \\
\hline Eu156 & $5.42 \mathrm{E}-11$ & $5.41 \mathrm{E}-11$ & $5.49 \mathrm{E}-11$ & $5.56 \mathrm{E}-11$ \\
\hline Gd152 & $3.05 \mathrm{E}-16$ & $3.05 \mathrm{E}-16$ & $3.05 \mathrm{E}-16$ & $3.05 \mathrm{E}-16$ \\
\hline Gd154 & $8.31 \mathrm{E}-16$ & $8.45 \mathrm{E}-16$ & $8.92 \mathrm{E}-16$ & $9.19 \mathrm{E}-16$ \\
\hline Gd155 & $3.18 \mathrm{E}-13$ & $3.20 \mathrm{E}-13$ & $3.24 \mathrm{E}-13$ & $3.25 \mathrm{E}-13$ \\
\hline Gd156 & $1.08 \mathrm{E}-12$ & $1.08 \mathrm{E}-12$ & $1.09 \mathrm{E}-12$ & $1.10 \mathrm{E}-12$ \\
\hline Gd157 & $1.99 \mathrm{E}-12$ & $2.00 \mathrm{E}-12$ & $2.05 \mathrm{E}-12$ & $2.10 \mathrm{E}-12$ \\
\hline Gd158 & $8.41 \mathrm{E}-12$ & $8.44 \mathrm{E}-12$ & $8.67 \mathrm{E}-12$ & $8.91 \mathrm{E}-12$ \\
\hline
\end{tabular}




\begin{tabular}{|l|l|l|l|l|}
\hline Gd160 & $1.15 \mathrm{E}-10$ & $1.16 \mathrm{E}-10$ & $1.20 \mathrm{E}-10$ & $1.23 \mathrm{E}-10$ \\
\hline Tb159 & $2.58 \mathrm{E}-13$ & $2.61 \mathrm{E}-13$ & $2.71 \mathrm{E}-13$ & $2.79 \mathrm{E}-13$ \\
\hline Tb160 & $3.42 \mathrm{E}-13$ & $3.50 \mathrm{E}-13$ & $5.38 \mathrm{E}-13$ & $5.72 \mathrm{E}-13$ \\
\hline$D y 160$ & $9.27 \mathrm{E}-16$ & $9.39 \mathrm{E}-16$ & $1.03 \mathrm{E}-15$ & $1.13 \mathrm{E}-15$ \\
\hline$D y 161$ & $4.46 \mathrm{E}-14$ & $4.52 \mathrm{E}-14$ & $4.75 \mathrm{E}-14$ & $4.94 \mathrm{E}-14$ \\
\hline$D y 162$ & $9.75 \mathrm{E}-14$ & $9.94 \mathrm{E}-14$ & $1.07 \mathrm{E}-13$ & $1.19 \mathrm{E}-13$ \\
\hline$D y 163$ & $1.51 \mathrm{E}-13$ & $1.56 \mathrm{E}-13$ & $1.68 \mathrm{E}-13$ & $1.97 \mathrm{E}-13$ \\
\hline$D y 164$ & $5.08 \mathrm{E}-13$ & $5.31 \mathrm{E}-13$ & $5.79 \mathrm{E}-13$ & $6.65 \mathrm{E}-13$ \\
\hline Ho165 & $5.94 \mathrm{E}-15$ & $6.42 \mathrm{E}-15$ & $7.48 \mathrm{E}-15$ & $9.40 \mathrm{E}-15$ \\
\hline Er166 & $9.00 \mathrm{E}-16$ & $9.40 \mathrm{E}-16$ & $1.01 \mathrm{E}-15$ & $1.08 \mathrm{E}-15$ \\
\hline Er167 & $7.29 \mathrm{E}-16$ & $7.96 \mathrm{E}-16$ & $9.14 \mathrm{E}-16$ & $1.06 \mathrm{E}-15$ \\
\hline Er168 & $2.15 \mathrm{E}-15$ & $2.57 \mathrm{E}-15$ & $3.22 \mathrm{E}-15$ & $4.35 \mathrm{E}-15$ \\
\hline
\end{tabular}




\section{Appendix 2: Detailed isotopic mass fraction output (discharged burn-up 50}

GWd/ tHM).

\begin{tabular}{|c|c|c|c|c|}
\hline \multicolumn{5}{|c|}{ Isotopic output mass vector } \\
\hline Isotope & ThPu & ThPuNp & ThPuNpAm & ThTRU \\
\hline IDs & $\mathrm{kg} / \mathrm{tIHM}$ & $\mathrm{kg} / \mathrm{tIHM}$ & $\mathrm{kg} / \mathrm{tIH} M$ & $\mathrm{~kg} / \mathrm{tIHM}$ \\
\hline $\mathrm{He} 4$ & $2.86 \mathrm{E}-02$ & -02 & $\mathrm{E}-02$ & $7.20 \mathrm{E}-02$ \\
\hline Ra228 & 2.82E-07 & $2.77 \mathrm{E}-07$ & $2.76 \mathrm{E}-07$ & $2.78 \mathrm{E}-07$ \\
\hline Th228 & $5.17 \mathrm{E}-04$ & $4.58 \mathrm{E}-04$ & $3.94 \mathrm{E}-04$ & 4.02E-04 \\
\hline Th229 & $1.96 \mathrm{E}-04$ & $1.77 \mathrm{E}-04$ & $1.58 \mathrm{E}-04$ & $1.60 \mathrm{E}-04$ \\
\hline Th230 & $1.56 \mathrm{E}-03$ & 1.5 & $1.62 \mathrm{E}-03$ & $1.63 \mathrm{E}-03$ \\
\hline Th231 & $2.75 \mathrm{E}-04$ & 2.68 & $2.58 \mathrm{E}-04$ & $2.61 \mathrm{E}-04$ \\
\hline Th232 & $8.65 \mathrm{E}+02$ & 8.40 & $8.03 \mathrm{E}+02$ & $8.06 \mathrm{E}+02$ \\
\hline Th233 & $3.82 \mathrm{E}-04$ & 3.49 & $3.15 \mathrm{E}-04$ & $3.19 \mathrm{E}-04$ \\
\hline Pa231 & $1.57 \mathrm{E}-01$ & 1.6 & $1.69 \mathrm{E}-01$ & $1.69 \mathrm{E}-01$ \\
\hline Pa233 & 7.02E-01 & 6.4 & $5.84 \mathrm{E}-01$ & $5.90 \mathrm{E}-01$ \\
\hline Pa234 & $6.74 \mathrm{E}-05$ & 5.6 & $4.57 \mathrm{E}-05$ & 4.66E-05 \\
\hline U232 & $6.50 \mathrm{E}-02$ & 5.68 & 4.79E-02 & 4.89E-02 \\
\hline U233 & $1.42 \mathrm{E}+01$ & $1.40 \mathrm{E}+01$ & $1.36 \mathrm{E}+01$ & $1.37 \mathrm{E}+01$ \\
\hline U234 & $1.31 \mathrm{E}+00$ & $1.22 \mathrm{E}+00$ & $1.11 \mathrm{E}+00$ & $1.13 \mathrm{E}+00$ \\
\hline U235 & $1.18 \mathrm{E}-01$ & $1.09 \mathrm{E}-01$ & $9.75 \mathrm{E}-02$ & $9.85 \mathrm{E}-02$ \\
\hline$U 236$ & $1.98 \mathrm{E}-02$ & $2.15 \mathrm{E}-02$ & $2.45 \mathrm{E}-02$ & $2.41 \mathrm{E}-02$ \\
\hline U237 & $3.83 \mathrm{E}-05$ & $3.89 \mathrm{E}-05$ & $4.05 \mathrm{E}-05$ & 4.02E-05 \\
\hline$U 238$ & $9.60 \mathrm{E}-05$ & $1.07 \mathrm{E}-04$ & $1.27 \mathrm{E}-04$ & $1.25 \mathrm{E}-04$ \\
\hline$N p 236$ & $1.80 \mathrm{E}-08$ & $1.21 \mathrm{E}-05$ & $2.08 \mathrm{E}-05$ & $1.99 \mathrm{E}-05$ \\
\hline$N p 236 M$ & 4.09E-10 & $1.63 \mathrm{E}-07$ & 2.19E-07 & $2.14 \mathrm{E}-07$ \\
\hline Np237 & $9.82 \mathrm{E}-03$ & $3.90 \mathrm{E}+00$ & $5.18 \mathrm{E}+00$ & $5.03 \mathrm{E}+00$ \\
\hline Np238 & $1.65 \mathrm{E}-05$ & $5.63 \mathrm{E}-03$ & $6.34 \mathrm{E}-03$ & $6.24 \mathrm{E}-03$ \\
\hline Np239 & $2.05 \mathrm{E}-06$ & $5.95 \mathrm{E}-06$ & $6.54 \mathrm{E}-06$ & $6.50 \mathrm{E}-06$ \\
\hline Pu236 & $1.93 \mathrm{E}-06$ & $4.53 \mathrm{E}-05$ & $6.21 \mathrm{E}-05$ & $6.06 \mathrm{E}-05$ \\
\hline Pu237 & $8.44 \mathrm{E}-06$ & $1.87 \mathrm{E}-05$ & $2.88 \mathrm{E}-05$ & $2.84 \mathrm{E}-05$ \\
\hline Pu238 & $2.92 \mathrm{E}+00$ & $6.10 \mathrm{E}+00$ & $8.98 \mathrm{E}+00$ & $8.82 \mathrm{E}+00$ \\
\hline Pu239 & $1.52 \mathrm{E}+01$ & $2.40 \mathrm{E}+01$ & $3.78 \mathrm{E}+01$ & $3.63 \mathrm{E}+01$ \\
\hline Pu240 & $2.14 \mathrm{E}+01$ & $2.71 \mathrm{E}+01$ & $3.53 \mathrm{E}+01$ & $3.44 \mathrm{E}+01$ \\
\hline Pu241 & $1.25 \mathrm{E}+01$ & $1.48 \mathrm{E}+01$ & $1.75 \mathrm{E}+01$ & $1.72 \mathrm{E}+01$ \\
\hline Pu242 & $9.37 \mathrm{E}+00$ & $1.05 \mathrm{E}+01$ & $1.27 \mathrm{E}+01$ & $1.24 \mathrm{E}+01$ \\
\hline Pu243 & $9.11 \mathrm{E}-04$ & 8.94E-04 & 8.91E-04 & $8.90 \mathrm{E}-04$ \\
\hline Pu244 & 8.88E-04 & $9.46 \mathrm{E}-04$ & $1.36 \mathrm{E}-03$ & $1.35 \mathrm{E}-03$ \\
\hline Am240 & 6.56E-08 & $8.66 \mathrm{E}-08$ & $2.22 \mathrm{E}-07$ & $2.17 \mathrm{E}-07$ \\
\hline$A m 241$ & $1.57 \mathrm{E}+00$ & $2.06 \mathrm{E}+00$ & $5.25 \mathrm{E}+00$ & $5.07 \mathrm{E}+00$ \\
\hline
\end{tabular}




\begin{tabular}{|c|c|c|c|c|}
\hline$A m 242$ & $1.56 \mathrm{E}-03$ & $1.63 \mathrm{E}-03$ & $3.15 \mathrm{E}-03$ & $3.10 \mathrm{E}-03$ \\
\hline$A m 242 M$ & $3.40 \mathrm{E}-02$ & $4.82 \mathrm{E}-02$ & $1.65 \mathrm{E}-01$ & $1.59 \mathrm{E}-01$ \\
\hline$A m 243$ & $2.30 \mathrm{E}+00$ & $2.43 \mathrm{E}+00$ & $3.81 \mathrm{E}+00$ & $3.75 \mathrm{E}+00$ \\
\hline Am244 & $7.38 \mathrm{E}-05$ & $6.91 \mathrm{E}-05$ & $8.96 \mathrm{E}-05$ & 8.94E-05 \\
\hline Am244M & $4.51 \mathrm{E}-05$ & $4.23 \mathrm{E}-05$ & $5.48 \mathrm{E}-05$ & $5.47 \mathrm{E}-05$ \\
\hline $\mathrm{Cm} 242$ & $2.87 \mathrm{E}-01$ & $3.00 \mathrm{E}-01$ & $6.35 \mathrm{E}-01$ & $6.27 \mathrm{E}-01$ \\
\hline $\mathrm{Cm} 243$ & $1.32 \mathrm{E}-02$ & $1.38 \mathrm{E}-02$ & $3.62 \mathrm{E}-02$ & $3.81 \mathrm{E}-02$ \\
\hline $\mathrm{Cm} 244$ & $1.19 \mathrm{E}+00$ & $1.14 \mathrm{E}+00$ & $2.15 \mathrm{E}+00$ & $2.65 \mathrm{E}+00$ \\
\hline $\mathrm{Cm} 245$ & $1.64 \mathrm{E}-01$ & $1.52 \mathrm{E}-01$ & $3.14 \mathrm{E}-01$ & $4.71 \mathrm{E}-01$ \\
\hline $\mathrm{Cm} 246$ & $1.07 \mathrm{E}-02$ & $7.90 \mathrm{E}-03$ & $1.61 \mathrm{E}-02$ & 4.07E-02 \\
\hline $\mathrm{Cm} 247$ & $1.90 \mathrm{E}-04$ & $1.35 \mathrm{E}-04$ & $3.09 \mathrm{E}-04$ & $1.55 \mathrm{E}-03$ \\
\hline $\mathrm{Cm} 248$ & $9.21 \mathrm{E}-06$ & $5.65 \mathrm{E}-06$ & $1.25 \mathrm{E}-05$ & $1.77 \mathrm{E}-04$ \\
\hline$B k 249$ & $1.79 \mathrm{E}-07$ & $1.11 \mathrm{E}-07$ & $2.58 \mathrm{E}-07$ & $5.12 \mathrm{E}-06$ \\
\hline$C f 249$ & $5.14 \mathrm{E}-08$ & $3.34 \mathrm{E}-08$ & $9.11 \mathrm{E}-08$ & $2.99 \mathrm{E}-06$ \\
\hline$Z n 70$ & $5.15 \mathrm{E}-06$ & $5.09 \mathrm{E}-06$ & $5.08 \mathrm{E}-06$ & $5.10 \mathrm{E}-06$ \\
\hline$Z n 72$ & $7.10 \mathrm{E}-08$ & $6.71 \mathrm{E}-08$ & $6.43 \mathrm{E}-08$ & $6.48 \mathrm{E}-08$ \\
\hline Ga71 & $1.25 \mathrm{E}-05$ & $1.21 \mathrm{E}-05$ & $1.19 \mathrm{E}-05$ & $1.20 \mathrm{E}-05$ \\
\hline Ge72 & $2.76 \mathrm{E}-05$ & $2.70 \mathrm{E}-05$ & $2.69 \mathrm{E}-05$ & $2.70 \mathrm{E}-05$ \\
\hline Ge73 & $6.01 \mathrm{E}-05$ & $5.80 \mathrm{E}-05$ & $5.65 \mathrm{E}-05$ & $5.68 \mathrm{E}-05$ \\
\hline Ge74 & $1.46 \mathrm{E}-04$ & $1.41 \mathrm{E}-04$ & $1.37 \mathrm{E}-04$ & $1.37 \mathrm{E}-04$ \\
\hline Ge76 & $7.00 \mathrm{E}-04$ & $6.73 \mathrm{E}-04$ & $6.48 \mathrm{E}-04$ & $6.51 \mathrm{E}-04$ \\
\hline Ge77 & 8.88E-07 & $8.38 \mathrm{E}-07$ & $7.95 \mathrm{E}-07$ & 7.99E-07 \\
\hline Ge78 & $2.77 \mathrm{E}-07$ & $2.63 \mathrm{E}-07$ & $2.50 \mathrm{E}-07$ & $2.51 \mathrm{E}-07$ \\
\hline Ge80 & $3.76 \mathrm{E}-10$ & $3.55 \mathrm{E}-10$ & $3.35 \mathrm{E}-10$ & $3.36 \mathrm{E}-10$ \\
\hline As 75 & $3.41 \mathrm{E}-04$ & $3.23 \mathrm{E}-04$ & $3.07 \mathrm{E}-04$ & 3.09E-04 \\
\hline As 77 & $3.18 \mathrm{E}-06$ & $3.00 \mathrm{E}-06$ & $2.85 \mathrm{E}-06$ & $2.86 \mathrm{E}-06$ \\
\hline As 78 & 2.99E-07 & $2.84 \mathrm{E}-07$ & $2.71 \mathrm{E}-07$ & $2.72 \mathrm{E}-07$ \\
\hline As 79 & $6.27 \mathrm{E}-08$ & $5.91 \mathrm{E}-08$ & $5.57 \mathrm{E}-08$ & $5.59 \mathrm{E}-08$ \\
\hline As 80 & $3.88 \mathrm{E}-10$ & $3.66 \mathrm{E}-10$ & $3.45 \mathrm{E}-10$ & $3.47 \mathrm{E}-10$ \\
\hline As 81 & $1.40 \mathrm{E}-09$ & $1.37 \mathrm{E}-09$ & $1.32 \mathrm{E}-09$ & $1.32 \mathrm{E}-09$ \\
\hline As 82 & $1.08 \mathrm{E}-10$ & $1.03 \mathrm{E}-10$ & $9.82 \mathrm{E}-11$ & $9.87 \mathrm{E}-11$ \\
\hline As $82 M$ & $7.88 \mathrm{E}-12$ & $7.48 \mathrm{E}-12$ & $7.08 \mathrm{E}-12$ & $7.11 \mathrm{E}-12$ \\
\hline As 83 & $2.26 \mathrm{E}-11$ & $2.11 \mathrm{E}-11$ & $1.97 \mathrm{E}-11$ & $1.99 \mathrm{E}-11$ \\
\hline $\mathrm{Se} 77$ & $1.45 \mathrm{E}-03$ & $1.41 \mathrm{E}-03$ & $1.38 \mathrm{E}-03$ & $1.38 \mathrm{E}-03$ \\
\hline $\mathrm{Se} 78$ & $3.80 \mathrm{E}-03$ & $3.70 \mathrm{E}-03$ & $3.60 \mathrm{E}-03$ & $3.60 \mathrm{E}-03$ \\
\hline $\mathrm{Se} 79$ & $8.48 \mathrm{E}-03$ & $8.22 \mathrm{E}-03$ & $7.95 \mathrm{E}-03$ & 7.97E-03 \\
\hline Se79M & $2.81 \mathrm{E}-08$ & $2.65 \mathrm{E}-08$ & $2.49 \mathrm{E}-08$ & $2.50 \mathrm{E}-08$ \\
\hline Se80 & $1.69 \mathrm{E}-02$ & $1.65 \mathrm{E}-02$ & $1.60 \mathrm{E}-02$ & $1.60 \mathrm{E}-02$ \\
\hline $\mathrm{Se} 81$ & 4.45E-07 & 4.33E-07 & $4.18 \mathrm{E}-07$ & 4.19E-07 \\
\hline Se81M & $1.12 \mathrm{E}-07$ & $1.13 \mathrm{E}-07$ & $1.14 \mathrm{E}-07$ & $1.13 \mathrm{E}-07$ \\
\hline
\end{tabular}




\begin{tabular}{|c|c|c|c|c|}
\hline $\mathrm{Se} 82$ & $4.67 \mathrm{E}-02$ & $4.55 \mathrm{E}-02$ & $4.41 \mathrm{E}-02$ & $4.42 \mathrm{E}-02$ \\
\hline $\mathrm{Se} 83$ & $1.18 \mathrm{E}-06$ & $1.11 \mathrm{E}-06$ & $1.03 \mathrm{E}-06$ & $1.04 \mathrm{E}-06$ \\
\hline Se $83 M$ & $2.27 \mathrm{E}-09$ & $2.16 \mathrm{E}-09$ & $2.05 \mathrm{E}-09$ & $2.05 \mathrm{E}-09$ \\
\hline $\mathrm{Se} 84$ & $1.98 \mathrm{E}-07$ & $1.85 \mathrm{E}-07$ & $1.73 \mathrm{E}-07$ & $1.74 \mathrm{E}-07$ \\
\hline $\mathrm{Se} 85$ & $4.05 \mathrm{E}-09$ & $3.84 \mathrm{E}-09$ & $3.62 \mathrm{E}-09$ & $3.63 \mathrm{E}-09$ \\
\hline Se86 & $1.15 \mathrm{E}-10$ & $1.10 \mathrm{E}-10$ & $1.04 \mathrm{E}-10$ & $1.04 \mathrm{E}-10$ \\
\hline $\mathrm{Se} 87$ & $9.80 \mathrm{E}-14$ & $9.42 \mathrm{E}-14$ & $9.01 \mathrm{E}-14$ & $9.05 \mathrm{E}-14$ \\
\hline$B r 79$ & $4.28 \mathrm{E}-06$ & $4.16 \mathrm{E}-06$ & $4.03 \mathrm{E}-06$ & $4.03 \mathrm{E}-06$ \\
\hline$B r 81$ & $3.03 \mathrm{E}-02$ & $2.98 \mathrm{E}-02$ & $2.92 \mathrm{E}-02$ & $2.92 \mathrm{E}-02$ \\
\hline Br82 & $3.00 \mathrm{E}-06$ & $2.86 \mathrm{E}-06$ & $2.69 \mathrm{E}-06$ & $2.70 \mathrm{E}-06$ \\
\hline$B r 83$ & $9.19 \mathrm{E}-06$ & $8.63 \mathrm{E}-06$ & $8.08 \mathrm{E}-06$ & $8.12 \mathrm{E}-06$ \\
\hline Br84 & $3.17 \mathrm{E}-06$ & $2.97 \mathrm{E}-06$ & $2.78 \mathrm{E}-06$ & $2.79 \mathrm{E}-06$ \\
\hline Br84M & $3.90 \mathrm{E}-08$ & $3.68 \mathrm{E}-08$ & $3.45 \mathrm{E}-08$ & $3.47 \mathrm{E}-08$ \\
\hline Br85 & $2.77 \mathrm{E}-07$ & $2.59 \mathrm{E}-07$ & $2.41 \mathrm{E}-07$ & $2.43 \mathrm{E}-07$ \\
\hline Br86 & $4.06 \mathrm{E}-08$ & $3.83 \mathrm{E}-08$ & $3.60 \mathrm{E}-08$ & $3.62 \mathrm{E}-08$ \\
\hline Br87 & $3.70 \mathrm{E}-08$ & $3.51 \mathrm{E}-08$ & 3.33E-08 & $3.35 \mathrm{E}-08$ \\
\hline$B r 88$ & $2.70 \mathrm{E}-10$ & $2.58 \mathrm{E}-10$ & $2.46 \mathrm{E}-10$ & $2.47 \mathrm{E}-10$ \\
\hline$B r 89$ & $1.48 \mathrm{E}-13$ & $1.44 \mathrm{E}-13$ & $1.39 \mathrm{E}-13$ & $1.40 \mathrm{E}-13$ \\
\hline$K r 82$ & $9.53 \mathrm{E}-04$ & $9.24 \mathrm{E}-04$ & 8.84E-04 & 8.89E-04 \\
\hline$K r 83$ & $6.30 \mathrm{E}-02$ & $6.16 \mathrm{E}-02$ & $6.00 \mathrm{E}-02$ & $6.01 \mathrm{E}-02$ \\
\hline Kr84 & $1.29 \mathrm{E}-01$ & $1.24 \mathrm{E}-01$ & $1.18 \mathrm{E}-01$ & $1.18 \mathrm{E}-01$ \\
\hline$K r 85$ & $3.51 \mathrm{E}-02$ & $3.35 \mathrm{E}-02$ & $3.18 \mathrm{E}-02$ & $3.20 \mathrm{E}-02$ \\
\hline Kr85M & $3.44 \mathrm{E}-05$ & $3.21 \mathrm{E}-05$ & $2.98 \mathrm{E}-05$ & $3.00 \mathrm{E}-05$ \\
\hline$K r 86$ & $2.01 \mathrm{E}-01$ & $1.93 \mathrm{E}-01$ & $1.85 \mathrm{E}-01$ & $1.86 \mathrm{E}-01$ \\
\hline Kr87 & $1.86 \mathrm{E}-05$ & $1.73 \mathrm{E}-05$ & $1.60 \mathrm{E}-05$ & $1.61 \mathrm{E}-05$ \\
\hline$K r 88$ & $5.37 \mathrm{E}-05$ & 4.99E-05 & $4.61 \mathrm{E}-05$ & $4.64 \mathrm{E}-05$ \\
\hline Kr89 & 7.23E-07 & $6.77 \mathrm{E}-07$ & $6.31 \mathrm{E}-07$ & $6.35 \mathrm{E}-07$ \\
\hline$K r 90$ & $1.48 \mathrm{E}-08$ & $1.39 \mathrm{E}-08$ & $1.31 \mathrm{E}-08$ & $1.31 \mathrm{E}-08$ \\
\hline$K r 91$ & $3.64 \mathrm{E}-12$ & $3.47 \mathrm{E}-12$ & $3.30 \mathrm{E}-12$ & $3.31 \mathrm{E}-12$ \\
\hline $\mathrm{Kr} 92$ & 7.44E-14 & $7.13 \mathrm{E}-14$ & $6.82 \mathrm{E}-14$ & $6.84 \mathrm{E}-14$ \\
\hline$R b 85$ & $1.13 \mathrm{E}-01$ & $1.09 \mathrm{E}-01$ & $1.04 \mathrm{E}-01$ & $1.04 \mathrm{E}-01$ \\
\hline$R b 87$ & $2.69 \mathrm{E}-01$ & $2.58 \mathrm{E}-01$ & $2.46 \mathrm{E}-01$ & $2.47 \mathrm{E}-01$ \\
\hline$R b 88$ & $5.92 \mathrm{E}-06$ & $5.49 \mathrm{E}-06$ & $5.06 \mathrm{E}-06$ & $5.10 \mathrm{E}-06$ \\
\hline$R b 89$ & $5.89 \mathrm{E}-06$ & $5.50 \mathrm{E}-06$ & $5.12 \mathrm{E}-06$ & $5.15 \mathrm{E}-06$ \\
\hline$R b 90$ & $6.42 \mathrm{E}-07$ & $6.04 \mathrm{E}-07$ & $5.67 \mathrm{E}-07$ & 5.70E-07 \\
\hline$R b 90 M$ & $3.66 \mathrm{E}-07$ & $3.43 \mathrm{E}-07$ & $3.21 \mathrm{E}-07$ & $3.23 \mathrm{E}-07$ \\
\hline$R b 91$ & $1.17 \mathrm{E}-07$ & $1.12 \mathrm{E}-07$ & $1.06 \mathrm{E}-07$ & $1.06 \mathrm{E}-07$ \\
\hline$R b 92$ & 7.39E-13 & $7.16 \mathrm{E}-13$ & $6.92 \mathrm{E}-13$ & $6.93 \mathrm{E}-13$ \\
\hline$R b 93$ & $7.26 \mathrm{E}-13$ & $7.14 \mathrm{E}-13$ & $7.00 \mathrm{E}-13$ & $7.00 \mathrm{E}-13$ \\
\hline$R b 94$ & $1.66 \mathrm{E}-13$ & $1.64 \mathrm{E}-13$ & $1.63 \mathrm{E}-13$ & $1.63 \mathrm{E}-13$ \\
\hline
\end{tabular}




\begin{tabular}{|c|c|c|c|c|}
\hline Sr86 & $6.00 \mathrm{E}-04$ & $5.74 \mathrm{E}-04$ & $5.47 \mathrm{E}-04$ & $5.51 \mathrm{E}-04$ \\
\hline Sr87 & $6.02 \mathrm{E}-06$ & $5.92 \mathrm{E}-06$ & $5.80 \mathrm{E}-06$ & $5.82 \mathrm{E}-06$ \\
\hline Sr88 & $3.56 \mathrm{E}-01$ & $3.41 \mathrm{E}-01$ & $3.24 \mathrm{E}-01$ & $3.25 \mathrm{E}-01$ \\
\hline Sr89 & $2.87 \mathrm{E}-02$ & $2.69 \mathrm{E}-02$ & $2.51 \mathrm{E}-02$ & $2.52 \mathrm{E}-02$ \\
\hline Sr90 & $4.94 \mathrm{E}-01$ & $4.77 \mathrm{E}-01$ & $4.58 \mathrm{E}-01$ & $4.59 \mathrm{E}-01$ \\
\hline $\operatorname{Sr} 91$ & $2.82 \mathrm{E}-04$ & $2.68 \mathrm{E}-04$ & $2.55 \mathrm{E}-04$ & $2.56 \mathrm{E}-04$ \\
\hline $\mathrm{Sr} 92$ & $8.50 \mathrm{E}-05$ & $8.20 \mathrm{E}-05$ & $7.88 \mathrm{E}-05$ & $7.90 \mathrm{E}-05$ \\
\hline $\mathrm{Sr} 93$ & $3.84 \mathrm{E}-06$ & $3.74 \mathrm{E}-06$ & $3.64 \mathrm{E}-06$ & $3.65 \mathrm{E}-06$ \\
\hline $\mathrm{Sr} 94$ & $2.64 \mathrm{E}-07$ & $2.60 \mathrm{E}-07$ & $2.55 \mathrm{E}-07$ & $2.55 \mathrm{E}-07$ \\
\hline Sr95 & $7.51 \mathrm{E}-09$ & $7.45 \mathrm{E}-09$ & 7.37E-09 & $7.36 \mathrm{E}-09$ \\
\hline Sr96 & $1.59 \mathrm{E}-13$ & $1.58 \mathrm{E}-13$ & $1.56 \mathrm{E}-13$ & $1.56 \mathrm{E}-13$ \\
\hline$Y 89$ & $4.10 \mathrm{E}-01$ & $3.95 \mathrm{E}-01$ & $3.78 \mathrm{E}-01$ & $3.80 \mathrm{E}-01$ \\
\hline$Y 90$ & $1.27 \mathrm{E}-04$ & $1.22 \mathrm{E}-04$ & $1.17 \mathrm{E}-04$ & $1.18 \mathrm{E}-04$ \\
\hline$Y 91$ & $4.03 \mathrm{E}-02$ & $3.86 \mathrm{E}-02$ & $3.67 \mathrm{E}-02$ & $3.68 \mathrm{E}-02$ \\
\hline$Y 91 M$ & $1.39 \mathrm{E}-05$ & $1.32 \mathrm{E}-05$ & $1.26 \mathrm{E}-05$ & $1.26 \mathrm{E}-05$ \\
\hline Y92 & $1.16 \mathrm{E}-04$ & $1.12 \mathrm{E}-04$ & $1.07 \mathrm{E}-04$ & $1.07 \mathrm{E}-04$ \\
\hline$Y 93$ & $3.91 \mathrm{E}-04$ & $3.81 \mathrm{E}-04$ & $3.69 \mathrm{E}-04$ & $3.69 \mathrm{E}-04$ \\
\hline$Y 94$ & $1.24 \mathrm{E}-05$ & $1.21 \mathrm{E}-05$ & $1.19 \mathrm{E}-05$ & $1.19 \mathrm{E}-05$ \\
\hline$Y 95$ & $6.61 \mathrm{E}-06$ & $6.55 \mathrm{E}-06$ & $6.47 \mathrm{E}-06$ & $6.46 \mathrm{E}-06$ \\
\hline$Y 96$ & 8.92E-13 & $8.87 \mathrm{E}-13$ & $8.78 \mathrm{E}-13$ & $8.78 \mathrm{E}-13$ \\
\hline$Y 96 M$ & $1.62 \mathrm{E}-11$ & $1.63 \mathrm{E}-11$ & $1.62 \mathrm{E}-11$ & $1.62 \mathrm{E}-11$ \\
\hline$Y 97$ & $6.29 \mathrm{E}-13$ & $6.29 \mathrm{E}-13$ & $6.27 \mathrm{E}-13$ & $6.26 \mathrm{E}-13$ \\
\hline$Y 97 M$ & $1.11 \mathrm{E}-13$ & $1.12 \mathrm{E}-13$ & $1.13 \mathrm{E}-13$ & $1.13 \mathrm{E}-13$ \\
\hline$Y 98$ & $6.66 \mathrm{E}-14$ & $6.73 \mathrm{E}-14$ & $6.76 \mathrm{E}-14$ & $6.75 \mathrm{E}-14$ \\
\hline$Y 98 M$ & $1.56 \mathrm{E}-13$ & $1.58 \mathrm{E}-13$ & $1.60 \mathrm{E}-13$ & $1.59 \mathrm{E}-13$ \\
\hline$Y 99$ & $1.67 \mathrm{E}-13$ & $1.68 \mathrm{E}-13$ & $1.68 \mathrm{E}-13$ & $1.68 \mathrm{E}-13$ \\
\hline $\operatorname{Zr} 90$ & $2.11 \mathrm{E}-02$ & $2.05 \mathrm{E}-02$ & $1.99 \mathrm{E}-02$ & $2.00 \mathrm{E}-02$ \\
\hline $\operatorname{Zr} 91$ & $5.30 \mathrm{E}-01$ & $5.17 \mathrm{E}-01$ & $5.02 \mathrm{E}-01$ & $5.03 \mathrm{E}-01$ \\
\hline $\mathrm{Zr92}$ & $6.64 \mathrm{E}-01$ & $6.51 \mathrm{E}-01$ & $6.35 \mathrm{E}-01$ & $6.36 \mathrm{E}-01$ \\
\hline $\mathrm{Zr93}$ & $7.96 \mathrm{E}-01$ & $7.85 \mathrm{E}-01$ & $7.71 \mathrm{E}-01$ & $7.71 \mathrm{E}-01$ \\
\hline $\mathrm{Zr} 94$ & $8.97 \mathrm{E}-01$ & $8.88 \mathrm{E}-01$ & $8.75 \mathrm{E}-01$ & $8.75 \mathrm{E}-01$ \\
\hline $\operatorname{Zr95}$ & $6.72 \mathrm{E}-02$ & $6.66 \mathrm{E}-02$ & $6.57 \mathrm{E}-02$ & $6.56 \mathrm{E}-02$ \\
\hline $\operatorname{Zr} 96$ & $9.95 \mathrm{E}-01$ & 9.92E-01 & $9.86 \mathrm{E}-01$ & $9.85 \mathrm{E}-01$ \\
\hline $\operatorname{Zr} 97$ & $7.92 \mathrm{E}-04$ & 7.93E-04 & $7.92 \mathrm{E}-04$ & 7.91E-04 \\
\hline $\operatorname{Zr} 98$ & $3.29 \mathrm{E}-08$ & 3.33E-08 & $3.35 \mathrm{E}-08$ & $3.35 \mathrm{E}-08$ \\
\hline $\mathrm{Zr} 99$ & $7.46 \mathrm{E}-13$ & $7.51 \mathrm{E}-13$ & $7.54 \mathrm{E}-13$ & $7.53 \mathrm{E}-13$ \\
\hline Zr100 & $3.91 \mathrm{E}-12$ & $3.96 \mathrm{E}-12$ & $4.00 \mathrm{E}-12$ & $3.99 \mathrm{E}-12$ \\
\hline$Z r 101$ & $4.48 \mathrm{E}-13$ & $4.50 \mathrm{E}-13$ & $4.50 \mathrm{E}-13$ & $4.50 \mathrm{E}-13$ \\
\hline Zr102 & $3.22 \mathrm{E}-13$ & $3.21 \mathrm{E}-13$ & $3.19 \mathrm{E}-13$ & $3.19 \mathrm{E}-13$ \\
\hline Zr103 & 4.61E-14 & $4.48 \mathrm{E}-14$ & 4.34E-14 & $4.35 \mathrm{E}-14$ \\
\hline
\end{tabular}




\begin{tabular}{|c|c|c|c|c|}
\hline $\mathrm{Nb94}$ & $3.04 \mathrm{E}-06$ & $3.11 \mathrm{E}-06$ & $3.22 \mathrm{E}-06$ & $3.21 \mathrm{E}-06$ \\
\hline $\mathrm{Nb95}$ & $3.66 \mathrm{E}-02$ & $3.62 \mathrm{E}-02$ & $3.58 \mathrm{E}-02$ & $3.57 \mathrm{E}-02$ \\
\hline Nb95M & $4.10 \mathrm{E}-05$ & $4.06 \mathrm{E}-05$ & $4.01 \mathrm{E}-05$ & $4.01 \mathrm{E}-05$ \\
\hline $\mathrm{Nb96}$ & $3.55 \mathrm{E}-06$ & $3.34 \mathrm{E}-06$ & $3.12 \mathrm{E}-06$ & $3.13 \mathrm{E}-06$ \\
\hline $\mathrm{Nb97}$ & $5.75 \mathrm{E}-05$ & $5.76 \mathrm{E}-05$ & $5.75 \mathrm{E}-05$ & $5.74 \mathrm{E}-05$ \\
\hline $\mathrm{Nb98}$ & $3.38 \mathrm{E}-09$ & $3.42 \mathrm{E}-09$ & $3.44 \mathrm{E}-09$ & $3.44 \mathrm{E}-09$ \\
\hline Nb98M & $2.54 \mathrm{E}-07$ & $2.57 \mathrm{E}-07$ & $2.61 \mathrm{E}-07$ & $2.60 \mathrm{E}-07$ \\
\hline $\mathrm{Nb99}$ & $1.89 \mathrm{E}-09$ & $1.93 \mathrm{E}-09$ & $1.97 \mathrm{E}-09$ & $1.96 \mathrm{E}-09$ \\
\hline Nb99M & $1.44 \mathrm{E}-07$ & $1.49 \mathrm{E}-07$ & $1.53 \mathrm{E}-07$ & $1.52 \mathrm{E}-07$ \\
\hline $\mathrm{Nb100}$ & $9.73 \mathrm{E}-13$ & $9.88 \mathrm{E}-13$ & $9.99 \mathrm{E}-13$ & $9.97 \mathrm{E}-13$ \\
\hline $\mathrm{Nb100M}$ & $1.18 \mathrm{E}-13$ & $1.22 \mathrm{E}-13$ & $1.27 \mathrm{E}-13$ & $1.27 \mathrm{E}-13$ \\
\hline $\mathrm{Nb101}$ & $4.74 \mathrm{E}-12$ & $4.84 \mathrm{E}-12$ & $4.93 \mathrm{E}-12$ & $4.92 \mathrm{E}-12$ \\
\hline $\mathrm{Nb102}$ & $2.69 \mathrm{E}-13$ & $2.72 \mathrm{E}-13$ & $2.76 \mathrm{E}-13$ & $2.75 \mathrm{E}-13$ \\
\hline $\mathrm{Nb103}$ & $3.34 \mathrm{E}-13$ & $3.39 \mathrm{E}-13$ & $3.44 \mathrm{E}-13$ & $3.43 \mathrm{E}-13$ \\
\hline $\mathrm{Nb104}$ & $3.24 \mathrm{E}-13$ & $3.23 \mathrm{E}-13$ & $3.21 \mathrm{E}-13$ & $3.21 \mathrm{E}-13$ \\
\hline $\mathrm{Nb105}$ & $1.47 \mathrm{E}-13$ & $1.46 \mathrm{E}-13$ & $1.45 \mathrm{E}-13$ & $1.45 \mathrm{E}-13$ \\
\hline Mo95 & 8.22E-01 & $8.20 \mathrm{E}-01$ & $8.15 \mathrm{E}-01$ & 8.14E-01 \\
\hline Mo96 & 4.71E-02 & 4.43E-02 & 4.10E-02 & 4.14E-02 \\
\hline Mo97 & $1.09 \mathrm{E}+00$ & $1.09 \mathrm{E}+00$ & $1.08 \mathrm{E}+00$ & $1.08 \mathrm{E}+00$ \\
\hline Mo98 & $1.15 \mathrm{E}+00$ & $1.15 \mathrm{E}+00$ & $1.15 \mathrm{E}+00$ & $1.15 \mathrm{E}+00$ \\
\hline Mo99 & $3.51 \mathrm{E}-03$ & $3.54 \mathrm{E}-03$ & $3.57 \mathrm{E}-03$ & $3.56 \mathrm{E}-03$ \\
\hline Mo100 & $1.33 \mathrm{E}+00$ & $1.34 \mathrm{E}+00$ & $1.34 \mathrm{E}+00$ & $1.34 \mathrm{E}+00$ \\
\hline Mo101 & $1.12 \mathrm{E}-05$ & $1.15 \mathrm{E}-05$ & $1.18 \mathrm{E}-05$ & $1.18 \mathrm{E}-05$ \\
\hline Mo102 & $8.35 \mathrm{E}-06$ & 8.61E-06 & $8.86 \mathrm{E}-06$ & $8.83 \mathrm{E}-06$ \\
\hline Mo103 & $3.03 \mathrm{E}-07$ & $3.16 \mathrm{E}-07$ & $3.29 \mathrm{E}-07$ & $3.28 \mathrm{E}-07$ \\
\hline Mo104 & $2.13 \mathrm{E}-07$ & $2.21 \mathrm{E}-07$ & $2.29 \mathrm{E}-07$ & $2.29 \mathrm{E}-07$ \\
\hline Mo105 & $3.88 \mathrm{E}-08$ & 4.02E-08 & $4.16 \mathrm{E}-08$ & 4.14E-08 \\
\hline Mo106 & $6.83 \mathrm{E}-12$ & $6.93 \mathrm{E}-12$ & $7.00 \mathrm{E}-12$ & $6.99 \mathrm{E}-12$ \\
\hline Mo107 & $2.63 \mathrm{E}-13$ & $2.59 \mathrm{E}-13$ & $2.55 \mathrm{E}-13$ & $2.55 \mathrm{E}-13$ \\
\hline Tc98 & 1.19E-05 & $1.22 \mathrm{E}-05$ & $1.27 \mathrm{E}-05$ & $1.27 \mathrm{E}-05$ \\
\hline Tc99 & $1.10 \mathrm{E}+00$ & $1.11 \mathrm{E}+00$ & $1.13 \mathrm{E}+00$ & $1.12 \mathrm{E}+00$ \\
\hline$T c 99 M$ & $2.83 \mathrm{E}-04$ & $2.86 \mathrm{E}-04$ & $2.88 \mathrm{E}-04$ & $2.87 \mathrm{E}-04$ \\
\hline Tc101 & $1.18 \mathrm{E}-05$ & $1.21 \mathrm{E}-05$ & $1.24 \mathrm{E}-05$ & $1.23 \mathrm{E}-05$ \\
\hline Tc102 & $6.56 \mathrm{E}-08$ & $6.76 \mathrm{E}-08$ & $6.95 \mathrm{E}-08$ & $6.93 \mathrm{E}-08$ \\
\hline Tc102M & $6.96 \mathrm{E}-09$ & $7.89 \mathrm{E}-09$ & $8.76 \mathrm{E}-09$ & $8.67 \mathrm{E}-09$ \\
\hline Tc103 & $4.88 \mathrm{E}-07$ & $5.10 \mathrm{E}-07$ & $5.31 \mathrm{E}-07$ & $5.29 \mathrm{E}-07$ \\
\hline Tc104 & $1.43 \mathrm{E}-05$ & $1.49 \mathrm{E}-05$ & $1.54 \mathrm{E}-05$ & $1.54 \mathrm{E}-05$ \\
\hline Tc105 & $4.78 \mathrm{E}-06$ & $5.01 \mathrm{E}-06$ & $5.23 \mathrm{E}-06$ & $5.22 \mathrm{E}-06$ \\
\hline Tc106 & 4.85E-08 & $4.99 \mathrm{E}-08$ & $5.13 \mathrm{E}-08$ & $5.11 \mathrm{E}-08$ \\
\hline Tc107 & $4.06 \mathrm{E}-09$ & 4.15E-09 & 4.28E-09 & 4.28E-09 \\
\hline
\end{tabular}




\begin{tabular}{|c|c|c|c|c|}
\hline Tc108 & $5.46 \mathrm{E}-13$ & $5.39 \mathrm{E}-13$ & $5.36 \mathrm{E}-13$ & $5.39 \mathrm{E}-13$ \\
\hline Tc109 & $4.08 \mathrm{E}-14$ & $4.03 \mathrm{E}-14$ & $3.99 \mathrm{E}-14$ & $4.00 \mathrm{E}-14$ \\
\hline Ru99 & $4.60 \mathrm{E}-05$ & $4.64 \mathrm{E}-05$ & $4.66 \mathrm{E}-05$ & $4.65 \mathrm{E}-05$ \\
\hline Ru100 & $1.72 \mathrm{E}-01$ & $1.61 \mathrm{E}-01$ & $1.48 \mathrm{E}-01$ & $1.49 \mathrm{E}-01$ \\
\hline Ru101 & $1.15 \mathrm{E}+00$ & $1.17 \mathrm{E}+00$ & $1.18 \mathrm{E}+00$ & $1.18 \mathrm{E}+00$ \\
\hline Ru102 & $1.31 \mathrm{E}+00$ & $1.32 \mathrm{E}+00$ & $1.34 \mathrm{E}+00$ & $1.33 \mathrm{E}+00$ \\
\hline Ru103 & 4.94E-02 & $5.14 \mathrm{E}-02$ & $5.33 \mathrm{E}-02$ & $5.31 \mathrm{E}-02$ \\
\hline Ru104 & $1.25 \mathrm{E}+00$ & $1.27 \mathrm{E}+00$ & $1.29 \mathrm{E}+00$ & $1.29 \mathrm{E}+00$ \\
\hline Ru105 & $1.91 \mathrm{E}-04$ & $2.00 \mathrm{E}-04$ & $2.09 \mathrm{E}-04$ & $2.08 \mathrm{E}-04$ \\
\hline Ru106 & $3.33 \mathrm{E}-01$ & $3.40 \mathrm{E}-01$ & $3.48 \mathrm{E}-01$ & $3.47 \mathrm{E}-01$ \\
\hline Ru107 & $1.42 \mathrm{E}-06$ & $1.46 \mathrm{E}-06$ & $1.52 \mathrm{E}-06$ & $1.52 \mathrm{E}-06$ \\
\hline$R u 108$ & $1.24 \mathrm{E}-06$ & $1.27 \mathrm{E}-06$ & $1.32 \mathrm{E}-06$ & $1.32 \mathrm{E}-06$ \\
\hline Ru109 & $1.47 \mathrm{E}-08$ & $1.51 \mathrm{E}-08$ & $1.55 \mathrm{E}-08$ & $1.55 \mathrm{E}-08$ \\
\hline Ru110 & $3.03 \mathrm{E}-11$ & $3.06 \mathrm{E}-11$ & $3.14 \mathrm{E}-11$ & $3.17 \mathrm{E}-11$ \\
\hline$R u 111$ & $5.08 \mathrm{E}-14$ & $5.11 \mathrm{E}-14$ & $5.23 \mathrm{E}-14$ & $5.30 \mathrm{E}-14$ \\
\hline$R h 103$ & $1.01 \mathrm{E}+00$ & $1.07 \mathrm{E}+00$ & $1.14 \mathrm{E}+00$ & $1.13 \mathrm{E}+00$ \\
\hline$R h 103 M$ & $4.86 \mathrm{E}-05$ & $5.05 \mathrm{E}-05$ & $5.23 \mathrm{E}-05$ & $5.21 \mathrm{E}-05$ \\
\hline Rh105 & $1.47 \mathrm{E}-03$ & $1.55 \mathrm{E}-03$ & $1.63 \mathrm{E}-03$ & $1.62 \mathrm{E}-03$ \\
\hline$R h 105 M$ & $1.44 \mathrm{E}-07$ & $1.51 \mathrm{E}-07$ & $1.58 \mathrm{E}-07$ & $1.58 \mathrm{E}-07$ \\
\hline Rh106 & $3.08 \mathrm{E}-07$ & $3.15 \mathrm{E}-07$ & $3.22 \mathrm{E}-07$ & $3.21 \mathrm{E}-07$ \\
\hline Rh106M & $2.89 \mathrm{E}-07$ & $2.51 \mathrm{E}-07$ & $2.14 \mathrm{E}-07$ & $2.16 \mathrm{E}-07$ \\
\hline Rh107 & $1.06 \mathrm{E}-05$ & $1.10 \mathrm{E}-05$ & $1.14 \mathrm{E}-05$ & $1.14 \mathrm{E}-05$ \\
\hline Rh108 & $8.10 \mathrm{E}-08$ & 8.30E-08 & 8.61E-08 & $8.62 \mathrm{E}-08$ \\
\hline$R h 108 M$ & 8.32E-09 & 9.19E-09 & $1.07 \mathrm{E}-08$ & $1.08 \mathrm{E}-08$ \\
\hline Rh109 & $1.93 \mathrm{E}-07$ & $1.97 \mathrm{E}-07$ & $2.04 \mathrm{E}-07$ & $2.05 \mathrm{E}-07$ \\
\hline Rh110 & $1.15 \mathrm{E}-11$ & $1.16 \mathrm{E}-11$ & $1.19 \mathrm{E}-11$ & $1.20 \mathrm{E}-11$ \\
\hline$R h 111$ & $1.16 \mathrm{E}-11$ & $1.17 \mathrm{E}-11$ & $1.26 \mathrm{E}-11$ & $1.29 \mathrm{E}-11$ \\
\hline$R h 112$ & $4.04 \mathrm{E}-14$ & $4.09 \mathrm{E}-14$ & $4.41 \mathrm{E}-14$ & $4.57 \mathrm{E}-14$ \\
\hline Pd104 & $2.80 \mathrm{E}-01$ & $2.44 \mathrm{E}-01$ & $1.94 \mathrm{E}-01$ & $1.98 \mathrm{E}-01$ \\
\hline$P d 105$ & $1.04 \mathrm{E}+00$ & $1.06 \mathrm{E}+00$ & $1.09 \mathrm{E}+00$ & $1.09 \mathrm{E}+00$ \\
\hline$P d 106$ & 7.17E-01 & $7.17 \mathrm{E}-01$ & $7.18 \mathrm{E}-01$ & $7.18 \mathrm{E}-01$ \\
\hline$P d 107$ & $7.11 \mathrm{E}-01$ & $7.22 \mathrm{E}-01$ & $7.38 \mathrm{E}-01$ & $7.37 \mathrm{E}-01$ \\
\hline$P d 108$ & $5.16 \mathrm{E}-01$ & $5.22 \mathrm{E}-01$ & $5.32 \mathrm{E}-01$ & $5.33 \mathrm{E}-01$ \\
\hline Pd109 & $2.84 \mathrm{E}-04$ & $2.85 \mathrm{E}-04$ & $2.89 \mathrm{E}-04$ & $2.90 \mathrm{E}-04$ \\
\hline$P d 110$ & $1.80 \mathrm{E}-01$ & $1.81 \mathrm{E}-01$ & $1.87 \mathrm{E}-01$ & $1.89 \mathrm{E}-01$ \\
\hline$P d 111$ & $1.36 \mathrm{E}-06$ & $1.37 \mathrm{E}-06$ & $1.46 \mathrm{E}-06$ & $1.50 \mathrm{E}-06$ \\
\hline$P d 111 M$ & $1.05 \mathrm{E}-07$ & $9.13 \mathrm{E}-08$ & $1.36 \mathrm{E}-07$ & $1.74 \mathrm{E}-07$ \\
\hline$P d 112$ & $3.35 \mathrm{E}-05$ & $3.36 \mathrm{E}-05$ & $3.69 \mathrm{E}-05$ & $3.89 \mathrm{E}-05$ \\
\hline$P d 113$ & $1.26 \mathrm{E}-08$ & $1.27 \mathrm{E}-08$ & $1.37 \mathrm{E}-08$ & $1.44 \mathrm{E}-08$ \\
\hline Pd114 & $1.56 \mathrm{E}-08$ & $1.59 \mathrm{E}-08$ & $1.73 \mathrm{E}-08$ & $1.81 \mathrm{E}-08$ \\
\hline
\end{tabular}




\begin{tabular}{|c|c|c|c|c|}
\hline $\operatorname{Pd} 115$ & $1.39 \mathrm{E}-10$ & $1.43 \mathrm{E}-10$ & $1.55 \mathrm{E}-10$ & $1.60 \mathrm{E}-10$ \\
\hline Pd116 & $1.75 \mathrm{E}-12$ & $1.83 \mathrm{E}-12$ & $1.95 \mathrm{E}-12$ & $1.97 \mathrm{E}-12$ \\
\hline $\operatorname{Ag} 109$ & $2.65 \mathrm{E}-01$ & $2.74 \mathrm{E}-01$ & $2.88 \mathrm{E}-01$ & $2.88 \mathrm{E}-01$ \\
\hline $\mathrm{Ag} 110 \mathrm{M}$ & $1.96 \mathrm{E}-03$ & $1.93 \mathrm{E}-03$ & $1.88 \mathrm{E}-03$ & $1.89 \mathrm{E}-03$ \\
\hline $\mathrm{Ag} 111$ & $6.88 \mathrm{E}-04$ & $6.87 \mathrm{E}-04$ & $7.25 \mathrm{E}-04$ & $7.47 \mathrm{E}-04$ \\
\hline $\mathrm{Ag} 111 \mathrm{M}$ & $6.33 \mathrm{E}-08$ & $6.36 \mathrm{E}-08$ & $6.77 \mathrm{E}-08$ & $6.97 \mathrm{E}-08$ \\
\hline $\operatorname{Ag} 112$ & $5.01 \mathrm{E}-06$ & $5.03 \mathrm{E}-06$ & $5.54 \mathrm{E}-06$ & $5.85 \mathrm{E}-06$ \\
\hline $\operatorname{Ag} 113$ & $3.85 \mathrm{E}-06$ & $3.83 \mathrm{E}-06$ & 4.12E-06 & $4.36 \mathrm{E}-06$ \\
\hline Ag113M & $1.52 \mathrm{E}-08$ & $1.52 \mathrm{E}-08$ & $1.64 \mathrm{E}-08$ & $1.73 \mathrm{E}-08$ \\
\hline $\operatorname{Ag} 115$ & $1.42 \mathrm{E}-07$ & $1.46 \mathrm{E}-07$ & $1.58 \mathrm{E}-07$ & $1.63 \mathrm{E}-07$ \\
\hline $\operatorname{Ag} 116$ & $1.17 \mathrm{E}-08$ & $1.23 \mathrm{E}-08$ & $1.32 \mathrm{E}-08$ & $1.33 \mathrm{E}-08$ \\
\hline $\mathrm{Ag} 117$ & 2.34E-09 & $2.49 \mathrm{E}-09$ & $2.66 \mathrm{E}-09$ & $2.68 \mathrm{E}-09$ \\
\hline Cd110 & $1.64 \mathrm{E}-01$ & $1.56 \mathrm{E}-01$ & $1.47 \mathrm{E}-01$ & $1.48 \mathrm{E}-01$ \\
\hline Cd111 & $8.66 \mathrm{E}-02$ & $8.65 \mathrm{E}-02$ & 8.97E-02 & $9.18 \mathrm{E}-02$ \\
\hline Cd112 & $3.87 \mathrm{E}-02$ & $3.89 \mathrm{E}-02$ & 4.14E-02 & $4.32 \mathrm{E}-02$ \\
\hline Cd113 & 8.04E-04 & $1.16 \mathrm{E}-03$ & $1.93 \mathrm{E}-03$ & $1.98 \mathrm{E}-03$ \\
\hline Cd113M & $1.18 \mathrm{E}-05$ & $1.66 \mathrm{E}-05$ & $2.71 \mathrm{E}-05$ & $2.77 \mathrm{E}-05$ \\
\hline Cd114 & $3.95 \mathrm{E}-02$ & $3.94 \mathrm{E}-02$ & $4.06 \mathrm{E}-02$ & 4.24E-02 \\
\hline Cd115 & $2.40 \mathrm{E}-05$ & $2.46 \mathrm{E}-05$ & $2.65 \mathrm{E}-05$ & $2.75 \mathrm{E}-05$ \\
\hline Cd115M & 3.09E-05 & $3.15 \mathrm{E}-05$ & $3.37 \mathrm{E}-05$ & $3.50 \mathrm{E}-05$ \\
\hline Cd116 & $1.09 \mathrm{E}-02$ & $1.12 \mathrm{E}-02$ & $1.16 \mathrm{E}-02$ & $1.17 \mathrm{E}-02$ \\
\hline Cd117 & $9.33 \mathrm{E}-07$ & $9.86 \mathrm{E}-07$ & $1.05 \mathrm{E}-06$ & $1.06 \mathrm{E}-06$ \\
\hline Cd117M & $3.98 \mathrm{E}-08$ & $4.38 \mathrm{E}-08$ & 4.73E-08 & 4.70E-08 \\
\hline Cd118 & $2.71 \mathrm{E}-07$ & $2.83 \mathrm{E}-07$ & $2.99 \mathrm{E}-07$ & $2.99 \mathrm{E}-07$ \\
\hline Cd119 & $7.55 \mathrm{E}-09$ & $7.83 \mathrm{E}-09$ & 8.17E-09 & $8.18 \mathrm{E}-09$ \\
\hline Cd119M & $1.26 \mathrm{E}-09$ & $1.33 \mathrm{E}-09$ & $1.41 \mathrm{E}-09$ & $1.40 \mathrm{E}-09$ \\
\hline Cd120 & $1.00 \mathrm{E}-09$ & $1.03 \mathrm{E}-09$ & $1.06 \mathrm{E}-09$ & $1.06 \mathrm{E}-09$ \\
\hline $\operatorname{In} 115$ & $4.38 \mathrm{E}-03$ & 4.95E-03 & $5.88 \mathrm{E}-03$ & $6.01 \mathrm{E}-03$ \\
\hline $\operatorname{In} 117$ & $1.68 \mathrm{E}-07$ & $1.78 \mathrm{E}-07$ & $1.90 \mathrm{E}-07$ & $1.91 \mathrm{E}-07$ \\
\hline In117M & $5.67 \mathrm{E}-07$ & 5.99E-07 & $6.39 \mathrm{E}-07$ & $6.43 \mathrm{E}-07$ \\
\hline $\operatorname{In} 119$ & 6.17E-09 & $6.45 \mathrm{E}-09$ & $6.76 \mathrm{E}-09$ & 6.76E-09 \\
\hline In119M & $5.20 \mathrm{E}-08$ & $5.38 \mathrm{E}-08$ & $5.60 \mathrm{E}-08$ & $5.61 \mathrm{E}-08$ \\
\hline $\operatorname{In} 121$ & $1.06 \mathrm{E}-10$ & $1.10 \mathrm{E}-10$ & $1.15 \mathrm{E}-10$ & $1.14 \mathrm{E}-10$ \\
\hline In $121 M$ & 5.12E-09 & $5.28 \mathrm{E}-09$ & $5.46 \mathrm{E}-09$ & $5.45 \mathrm{E}-09$ \\
\hline Sn115 & $5.10 \mathrm{E}-04$ & $5.22 \mathrm{E}-04$ & $5.49 \mathrm{E}-04$ & $5.64 \mathrm{E}-04$ \\
\hline Sn116 & $3.53 \mathrm{E}-03$ & $3.32 \mathrm{E}-03$ & $3.08 \mathrm{E}-03$ & $3.19 \mathrm{E}-03$ \\
\hline Sn117 & $9.98 \mathrm{E}-03$ & $1.03 \mathrm{E}-02$ & $1.06 \mathrm{E}-02$ & $1.07 \mathrm{E}-02$ \\
\hline Sn118 & 8.30E-03 & $8.51 \mathrm{E}-03$ & $8.80 \mathrm{E}-03$ & $8.82 \mathrm{E}-03$ \\
\hline Sn119 & 8.23E-03 & $8.43 \mathrm{E}-03$ & 8.67E-03 & $8.68 \mathrm{E}-03$ \\
\hline Sn 120 & 8.38E-03 & $8.55 \mathrm{E}-03$ & $8.78 \mathrm{E}-03$ & $8.78 \mathrm{E}-03$ \\
\hline
\end{tabular}




\begin{tabular}{|c|c|c|c|c|}
\hline Sn 121 & $9.80 \mathrm{E}-06$ & $1.02 \mathrm{E}-05$ & $1.06 \mathrm{E}-05$ & $1.06 \mathrm{E}-05$ \\
\hline Sn121M & $5.65 \mathrm{E}-04$ & 5.83E-04 & $6.04 \mathrm{E}-04$ & $6.03 \mathrm{E}-04$ \\
\hline Sn122 & $1.11 \mathrm{E}-02$ & $1.13 \mathrm{E}-02$ & $1.16 \mathrm{E}-02$ & $1.16 \mathrm{E}-02$ \\
\hline Sn123 & $6.81 \mathrm{E}-04$ & $6.91 \mathrm{E}-04$ & $7.06 \mathrm{E}-04$ & $7.07 \mathrm{E}-04$ \\
\hline Sn $123 M$ & $2.01 \mathrm{E}-07$ & $2.02 \mathrm{E}-07$ & $2.04 \mathrm{E}-07$ & $2.04 \mathrm{E}-07$ \\
\hline Sn 124 & $1.80 \mathrm{E}-02$ & $1.83 \mathrm{E}-02$ & $1.87 \mathrm{E}-02$ & $1.86 \mathrm{E}-02$ \\
\hline Sn $125 M$ & 4.93E-08 & $5.01 \mathrm{E}-08$ & $5.11 \mathrm{E}-08$ & $5.11 \mathrm{E}-08$ \\
\hline Sn126 & $4.66 \mathrm{E}-02$ & 4.69E-02 & $4.74 \mathrm{E}-02$ & $4.74 \mathrm{E}-02$ \\
\hline Sn127 & 7.49E-06 & 7.53E-06 & $7.57 \mathrm{E}-06$ & $7.57 \mathrm{E}-06$ \\
\hline Sn $127 M$ & $6.19 \mathrm{E}-08$ & $6.23 \mathrm{E}-08$ & $6.26 \mathrm{E}-08$ & $6.26 \mathrm{E}-08$ \\
\hline Sn 128 & $6.36 \mathrm{E}-06$ & $6.42 \mathrm{E}-06$ & $6.47 \mathrm{E}-06$ & $6.47 \mathrm{E}-06$ \\
\hline Sn129 & $1.67 \mathrm{E}-07$ & $1.67 \mathrm{E}-07$ & $1.67 \mathrm{E}-07$ & $1.67 \mathrm{E}-07$ \\
\hline Sn129M & $2.98 \mathrm{E}-07$ & 2.99E-07 & $3.01 \mathrm{E}-07$ & $3.00 \mathrm{E}-07$ \\
\hline Sn130 & $2.81 \mathrm{E}-07$ & $2.82 \mathrm{E}-07$ & $2.82 \mathrm{E}-07$ & $2.82 \mathrm{E}-07$ \\
\hline Sn131 & $1.90 \mathrm{E}-08$ & $1.89 \mathrm{E}-08$ & $1.87 \mathrm{E}-08$ & $1.87 \mathrm{E}-08$ \\
\hline Sn132 & 7.88E-09 & 8.14E-09 & 8.31E-09 & 8.27E-09 \\
\hline$S b 121$ & $8.10 \mathrm{E}-03$ & 8.33E-03 & $8.61 \mathrm{E}-03$ & $8.58 \mathrm{E}-03$ \\
\hline Sb122 & $4.65 \mathrm{E}-06$ & 4.47E-06 & 4.23E-06 & $4.25 \mathrm{E}-06$ \\
\hline Sb123 & $1.07 \mathrm{E}-02$ & $1.09 \mathrm{E}-02$ & $1.11 \mathrm{E}-02$ & $1.11 \mathrm{E}-02$ \\
\hline Sb124 & $6.26 \mathrm{E}-05$ & $6.01 \mathrm{E}-05$ & $5.75 \mathrm{E}-05$ & $5.79 \mathrm{E}-05$ \\
\hline Sb125 & $1.63 \mathrm{E}-02$ & $1.65 \mathrm{E}-02$ & $1.68 \mathrm{E}-02$ & $1.68 \mathrm{E}-02$ \\
\hline$S b 126$ & $7.46 \mathrm{E}-06$ & $7.57 \mathrm{E}-06$ & $7.70 \mathrm{E}-06$ & $7.70 \mathrm{E}-06$ \\
\hline$S b 127$ & 4.63E-04 & 4.66E-04 & 4.70E-04 & 4.70E-04 \\
\hline Sb128 & $6.45 \mathrm{E}-05$ & $6.51 \mathrm{E}-05$ & $6.56 \mathrm{E}-05$ & $6.55 \mathrm{E}-05$ \\
\hline$S b 128 M$ & $8.06 \mathrm{E}-08$ & $8.12 \mathrm{E}-08$ & $8.21 \mathrm{E}-08$ & $8.20 \mathrm{E}-08$ \\
\hline Sb129 & $6.47 \mathrm{E}-05$ & $6.50 \mathrm{E}-05$ & $6.53 \mathrm{E}-05$ & $6.53 \mathrm{E}-05$ \\
\hline$S b 130$ & $7.41 \mathrm{E}-06$ & 7.52E-06 & 7.63E-06 & 7.61E-06 \\
\hline Sb130M & $1.14 \mathrm{E}-06$ & $1.14 \mathrm{E}-06$ & $1.15 \mathrm{E}-06$ & $1.15 \mathrm{E}-06$ \\
\hline Sb131 & $1.12 \mathrm{E}-05$ & $1.12 \mathrm{E}-05$ & $1.13 \mathrm{E}-05$ & $1.13 \mathrm{E}-05$ \\
\hline$S b 132$ & $6.74 \mathrm{E}-07$ & $6.99 \mathrm{E}-07$ & $7.17 \mathrm{E}-07$ & 7.14E-07 \\
\hline$S b 132 M$ & 4.40E-07 & 4.39E-07 & $4.38 \mathrm{E}-07$ & $4.38 \mathrm{E}-07$ \\
\hline$S b 133$ & $5.06 \mathrm{E}-07$ & $5.01 \mathrm{E}-07$ & $4.94 \mathrm{E}-07$ & 4.94E-07 \\
\hline$S b 134 M$ & $4.96 \mathrm{E}-12$ & $4.89 \mathrm{E}-12$ & $4.79 \mathrm{E}-12$ & $4.79 \mathrm{E}-12$ \\
\hline $\mathrm{Te} 122$ & 7.79E-04 & $7.51 \mathrm{E}-04$ & $7.15 \mathrm{E}-04$ & $7.20 \mathrm{E}-04$ \\
\hline $\mathrm{Te} 124$ & 4.84E-04 & $4.68 \mathrm{E}-04$ & $4.52 \mathrm{E}-04$ & 4.55E-04 \\
\hline $\mathrm{Te} 125$ & $8.70 \mathrm{E}-03$ & $8.79 \mathrm{E}-03$ & $8.90 \mathrm{E}-03$ & $8.90 \mathrm{E}-03$ \\
\hline $\mathrm{Te} 125 \mathrm{M}$ & $2.10 \mathrm{E}-04$ & $2.13 \mathrm{E}-04$ & $2.17 \mathrm{E}-04$ & $2.17 \mathrm{E}-04$ \\
\hline $\mathrm{Te} 126$ & $9.58 \mathrm{E}-04$ & $9.69 \mathrm{E}-04$ & $9.85 \mathrm{E}-04$ & $9.85 \mathrm{E}-04$ \\
\hline $\mathrm{Te} 127$ & 4.63E-05 & $4.68 \mathrm{E}-05$ & 4.72E-05 & 4.72E-05 \\
\hline $\mathrm{Te} 127 \mathrm{M}$ & $5.02 \mathrm{E}-04$ & $5.35 \mathrm{E}-04$ & 5.67E-04 & $5.65 \mathrm{E}-04$ \\
\hline
\end{tabular}




\begin{tabular}{|c|c|c|c|c|}
\hline $\mathrm{Te} 128$ & $1.79 \mathrm{E}-01$ & $1.80 \mathrm{E}-01$ & $1.80 \mathrm{E}-01$ & $1.80 \mathrm{E}-01$ \\
\hline $\mathrm{Te} 129 \mathrm{M}$ & $6.03 \mathrm{E}-05$ & $5.27 \mathrm{E}-05$ & $4.55 \mathrm{E}-05$ & 4.63E-05 \\
\hline $\mathrm{Te} 130$ & 5.99E-01 & $6.02 \mathrm{E}-01$ & $6.03 \mathrm{E}-01$ & $6.02 \mathrm{E}-01$ \\
\hline Te131 & $1.50 \mathrm{E}-05$ & $1.51 \mathrm{E}-05$ & $1.53 \mathrm{E}-05$ & $1.52 \mathrm{E}-05$ \\
\hline $\mathrm{Te} 131 \mathrm{M}$ & $2.46 \mathrm{E}-04$ & $2.47 \mathrm{E}-04$ & $2.48 \mathrm{E}-04$ & $2.48 \mathrm{E}-04$ \\
\hline Te132 & $4.54 \mathrm{E}-03$ & $4.57 \mathrm{E}-03$ & $4.59 \mathrm{E}-03$ & $4.58 \mathrm{E}-03$ \\
\hline Te133 & 8.69E-06 & $8.71 \mathrm{E}-06$ & $8.70 \mathrm{E}-06$ & $8.68 \mathrm{E}-06$ \\
\hline Te133M & $3.25 \mathrm{E}-05$ & $3.25 \mathrm{E}-05$ & $3.24 \mathrm{E}-05$ & $3.23 \mathrm{E}-05$ \\
\hline Te134 & $4.40 \mathrm{E}-05$ & 4.39E-05 & 4.37E-05 & $4.36 \mathrm{E}-05$ \\
\hline Te135 & 3.07E-09 & $3.05 \mathrm{E}-09$ & $3.01 \mathrm{E}-09$ & $3.01 \mathrm{E}-09$ \\
\hline Te136 & $7.93 \mathrm{E}-10$ & $7.66 \mathrm{E}-10$ & 7.37E-10 & $7.38 \mathrm{E}-10$ \\
\hline Te137 & $6.35 \mathrm{E}-14$ & $6.16 \mathrm{E}-14$ & $5.93 \mathrm{E}-14$ & $5.93 \mathrm{E}-14$ \\
\hline$I 127$ & $1.08 \mathrm{E}-01$ & $1.08 \mathrm{E}-01$ & $1.09 \mathrm{E}-01$ & $1.09 \mathrm{E}-01$ \\
\hline$I 128$ & $3.61 \mathrm{E}-07$ & $3.47 \mathrm{E}-07$ & $3.29 \mathrm{E}-07$ & $3.32 \mathrm{E}-07$ \\
\hline$I 129$ & $3.33 \mathrm{E}-01$ & $3.34 \mathrm{E}-01$ & $3.35 \mathrm{E}-01$ & $3.35 \mathrm{E}-01$ \\
\hline$I 130$ & 8.61E-06 & $7.57 \mathrm{E}-06$ & $6.63 \mathrm{E}-06$ & $6.71 \mathrm{E}-06$ \\
\hline$I 130 M$ & 5.62E-08 & 4.92E-08 & 4.29E-08 & 4.34E-08 \\
\hline$I 132$ & $1.40 \mathrm{E}-04$ & $1.41 \mathrm{E}-04$ & $1.42 \mathrm{E}-04$ & $1.42 \mathrm{E}-04$ \\
\hline$I 133$ & $1.69 \mathrm{E}-03$ & $1.70 \mathrm{E}-03$ & $1.71 \mathrm{E}-03$ & $1.71 \mathrm{E}-03$ \\
\hline$I 133 M$ & $2.01 \mathrm{E}-12$ & $2.13 \mathrm{E}-12$ & $2.27 \mathrm{E}-12$ & $2.26 \mathrm{E}-12$ \\
\hline$I 134$ & 7.82E-05 & $7.86 \mathrm{E}-05$ & 7.89E-05 & $7.88 \mathrm{E}-05$ \\
\hline$I 134 M$ & 4.44E-07 & 4.57E-07 & $4.71 \mathrm{E}-07$ & 4.70E-07 \\
\hline$I 135$ & $5.16 \mathrm{E}-04$ & $5.21 \mathrm{E}-04$ & $5.23 \mathrm{E}-04$ & $5.23 \mathrm{E}-04$ \\
\hline$I 136$ & $3.23 \mathrm{E}-07$ & $3.17 \mathrm{E}-07$ & $3.11 \mathrm{E}-07$ & $3.11 \mathrm{E}-07$ \\
\hline$I 136 M$ & 5.12E-08 & $5.16 \mathrm{E}-08$ & $5.20 \mathrm{E}-08$ & 5.19E-08 \\
\hline$I 137$ & $1.02 \mathrm{E}-08$ & $1.01 \mathrm{E}-08$ & $1.01 \mathrm{E}-08$ & $1.01 \mathrm{E}-08$ \\
\hline 1138 & $1.03 \mathrm{E}-12$ & $1.03 \mathrm{E}-12$ & $1.02 \mathrm{E}-12$ & $1.02 \mathrm{E}-12$ \\
\hline$I 139$ & $1.19 \mathrm{E}-13$ & $1.16 \mathrm{E}-13$ & $1.13 \mathrm{E}-13$ & $1.13 \mathrm{E}-13$ \\
\hline $\mathrm{Xe} 128$ & $9.46 \mathrm{E}-03$ & $9.14 \mathrm{E}-03$ & $8.73 \mathrm{E}-03$ & 8.79E-03 \\
\hline Xe130 & 8.49E-03 & $7.70 \mathrm{E}-03$ & $6.94 \mathrm{E}-03$ & $7.00 \mathrm{E}-03$ \\
\hline Xe131 & 7.25E-01 & $7.48 \mathrm{E}-01$ & 7.74E-01 & $7.70 \mathrm{E}-01$ \\
\hline Xe132 & $1.70 \mathrm{E}+00$ & $1.69 \mathrm{E}+00$ & $1.66 \mathrm{E}+00$ & $1.66 \mathrm{E}+00$ \\
\hline Xe133 & 9.89E-03 & $9.95 \mathrm{E}-03$ & $9.99 \mathrm{E}-03$ & $9.97 \mathrm{E}-03$ \\
\hline Xe133M & 6.32E-05 & $6.36 \mathrm{E}-05$ & $6.39 \mathrm{E}-05$ & $6.38 \mathrm{E}-05$ \\
\hline Xe134 & $2.16 \mathrm{E}+00$ & $2.16 \mathrm{E}+00$ & $2.16 \mathrm{E}+00$ & $2.16 \mathrm{E}+00$ \\
\hline Xe135 & 4.89E-04 & $5.64 \mathrm{E}-04$ & $6.41 \mathrm{E}-04$ & $6.35 \mathrm{E}-04$ \\
\hline Xe135M & $3.62 \mathrm{E}-06$ & $3.67 \mathrm{E}-06$ & $3.71 \mathrm{E}-06$ & $3.71 \mathrm{E}-06$ \\
\hline Xe136 & $2.72 \mathrm{E}+00$ & $2.59 \mathrm{E}+00$ & $2.44 \mathrm{E}+00$ & $2.45 \mathrm{E}+00$ \\
\hline Xe137 & $3.75 \mathrm{E}-06$ & $3.74 \mathrm{E}-06$ & $3.72 \mathrm{E}-06$ & $3.72 \mathrm{E}-06$ \\
\hline Xe138 & $1.52 \mathrm{E}-05$ & $1.52 \mathrm{E}-05$ & $1.51 \mathrm{E}-05$ & $1.51 \mathrm{E}-05$ \\
\hline
\end{tabular}




\begin{tabular}{|c|c|c|c|c|}
\hline Xe139 & $7.75 \mathrm{E}-08$ & $7.66 \mathrm{E}-08$ & $7.56 \mathrm{E}-08$ & $7.57 \mathrm{E}-08$ \\
\hline Xe140 & $3.78 \mathrm{E}-10$ & $3.70 \mathrm{E}-10$ & $3.62 \mathrm{E}-10$ & $3.62 \mathrm{E}-10$ \\
\hline Xe141 & $1.49 \mathrm{E}-13$ & $1.45 \mathrm{E}-13$ & $1.41 \mathrm{E}-13$ & $1.41 \mathrm{E}-13$ \\
\hline Cs133 & $1.61 \mathrm{E}+00$ & $1.64 \mathrm{E}+00$ & $1.66 \mathrm{E}+00$ & $1.66 \mathrm{E}+00$ \\
\hline Cs134 & $1.95 \mathrm{E}-01$ & $1.82 \mathrm{E}-01$ & $1.69 \mathrm{E}-01$ & $1.70 \mathrm{E}-01$ \\
\hline Cs $134 M$ & $6.97 \mathrm{E}-06$ & $6.46 \mathrm{E}-06$ & $5.92 \mathrm{E}-06$ & $5.96 \mathrm{E}-06$ \\
\hline Cs 135 & $1.39 \mathrm{E}+00$ & $1.53 \mathrm{E}+00$ & $1.67 \mathrm{E}+00$ & $1.66 \mathrm{E}+00$ \\
\hline Cs $135 M$ & $2.44 \mathrm{E}-07$ & $2.64 \mathrm{E}-07$ & $2.85 \mathrm{E}-07$ & $2.84 \mathrm{E}-07$ \\
\hline Cs 136 & $1.30 \mathrm{E}-03$ & $1.33 \mathrm{E}-03$ & $1.35 \mathrm{E}-03$ & $1.35 \mathrm{E}-03$ \\
\hline Cs137 & $1.86 \mathrm{E}+00$ & $1.86 \mathrm{E}+00$ & $1.85 \mathrm{E}+00$ & $1.85 \mathrm{E}+00$ \\
\hline Cs138 & $4.39 \mathrm{E}-05$ & $4.39 \mathrm{E}-05$ & $4.38 \mathrm{E}-05$ & $4.38 \mathrm{E}-05$ \\
\hline Cs $138 M$ & $1.96 \mathrm{E}-07$ & $1.97 \mathrm{E}-07$ & $2.00 \mathrm{E}-07$ & $2.00 \mathrm{E}-07$ \\
\hline Cs139 & $1.06 \mathrm{E}-05$ & $1.05 \mathrm{E}-05$ & $1.04 \mathrm{E}-05$ & $1.04 \mathrm{E}-05$ \\
\hline Cs 140 & $3.65 \mathrm{E}-07$ & $3.60 \mathrm{E}-07$ & $3.55 \mathrm{E}-07$ & $3.55 \mathrm{E}-07$ \\
\hline Cs 141 & $1.55 \mathrm{E}-08$ & $1.53 \mathrm{E}-08$ & $1.52 \mathrm{E}-08$ & $1.52 \mathrm{E}-08$ \\
\hline Cs 142 & $3.66 \mathrm{E}-13$ & $3.60 \mathrm{E}-13$ & $3.52 \mathrm{E}-13$ & $3.53 \mathrm{E}-13$ \\
\hline Cs 143 & $1.89 \mathrm{E}-13$ & $1.86 \mathrm{E}-13$ & $1.82 \mathrm{E}-13$ & $1.82 \mathrm{E}-13$ \\
\hline Ba134 & $9.28 \mathrm{E}-02$ & 8.65E-02 & 8.02E-02 & 8.07E-02 \\
\hline Ba135 & $7.33 \mathrm{E}-04$ & $6.59 \mathrm{E}-04$ & $5.87 \mathrm{E}-04$ & $5.95 \mathrm{E}-04$ \\
\hline Ba136 & $6.40 \mathrm{E}-02$ & $6.51 \mathrm{E}-02$ & $6.60 \mathrm{E}-02$ & $6.62 \mathrm{E}-02$ \\
\hline Ba137 & $8.28 \mathrm{E}-02$ & 8.27E-02 & $8.25 \mathrm{E}-02$ & $8.25 \mathrm{E}-02$ \\
\hline Ba137M & $2.85 \mathrm{E}-07$ & $2.84 \mathrm{E}-07$ & $2.84 \mathrm{E}-07$ & $2.84 \mathrm{E}-07$ \\
\hline Ba138 & $1.85 \mathrm{E}+00$ & $1.84 \mathrm{E}+00$ & $1.84 \mathrm{E}+00$ & $1.84 \mathrm{E}+00$ \\
\hline Ba139 & $1.09 \mathrm{E}-04$ & $1.08 \mathrm{E}-04$ & $1.07 \mathrm{E}-04$ & $1.07 \mathrm{E}-04$ \\
\hline Ba140 & $2.34 \mathrm{E}-02$ & $2.31 \mathrm{E}-02$ & $2.29 \mathrm{E}-02$ & $2.29 \mathrm{E}-02$ \\
\hline Ba141 & $2.07 \mathrm{E}-05$ & $2.05 \mathrm{E}-05$ & $2.03 \mathrm{E}-05$ & $2.03 \mathrm{E}-05$ \\
\hline Ba142 & $1.07 \mathrm{E}-05$ & $1.05 \mathrm{E}-05$ & $1.04 \mathrm{E}-05$ & $1.04 \mathrm{E}-05$ \\
\hline Ba143 & 1.07E-09 & $1.06 \mathrm{E}-09$ & $1.05 \mathrm{E}-09$ & $1.05 \mathrm{E}-09$ \\
\hline Ba144 & $1.61 \mathrm{E}-10$ & $1.57 \mathrm{E}-10$ & $1.53 \mathrm{E}-10$ & $1.53 \mathrm{E}-10$ \\
\hline Ba145 & $6.15 \mathrm{E}-13$ & $5.95 \mathrm{E}-13$ & $5.73 \mathrm{E}-13$ & $5.75 \mathrm{E}-13$ \\
\hline Ba146 & $1.27 \mathrm{E}-13$ & $1.22 \mathrm{E}-13$ & $1.16 \mathrm{E}-13$ & $1.16 \mathrm{E}-13$ \\
\hline$L a 138$ & $3.03 \mathrm{E}-05$ & $3.11 \mathrm{E}-05$ & $3.28 \mathrm{E}-05$ & $3.29 \mathrm{E}-05$ \\
\hline La139 & $1.75 \mathrm{E}+00$ & $1.74 \mathrm{E}+00$ & $1.74 \mathrm{E}+00$ & $1.74 \mathrm{E}+00$ \\
\hline La140 & $3.27 \mathrm{E}-03$ & $3.23 \mathrm{E}-03$ & $3.19 \mathrm{E}-03$ & $3.19 \mathrm{E}-03$ \\
\hline La141 & $2.85 \mathrm{E}-04$ & $2.82 \mathrm{E}-04$ & $2.79 \mathrm{E}-04$ & $2.80 \mathrm{E}-04$ \\
\hline La142 & $1.08 \mathrm{E}-04$ & $1.07 \mathrm{E}-04$ & $1.05 \mathrm{E}-04$ & $1.05 \mathrm{E}-04$ \\
\hline La143 & $1.44 \mathrm{E}-05$ & $1.42 \mathrm{E}-05$ & $1.40 \mathrm{E}-05$ & $1.40 \mathrm{E}-05$ \\
\hline La144 & $1.23 \mathrm{E}-07$ & $1.21 \mathrm{E}-07$ & $1.19 \mathrm{E}-07$ & $1.19 \mathrm{E}-07$ \\
\hline La145 & $1.30 \mathrm{E}-08$ & $1.29 \mathrm{E}-08$ & $1.29 \mathrm{E}-08$ & $1.29 \mathrm{E}-08$ \\
\hline La146 & $9.28 \mathrm{E}-13$ & $9.07 \mathrm{E}-13$ & $8.85 \mathrm{E}-13$ & $8.87 \mathrm{E}-13$ \\
\hline
\end{tabular}




\begin{tabular}{|c|c|c|c|c|}
\hline La147 & 4.19E-13 & 4.10E-13 & $4.00 \mathrm{E}-13$ & $4.01 \mathrm{E}-13$ \\
\hline La148 & 4.94E-14 & 4.72E-14 & $4.51 \mathrm{E}-14$ & $4.53 \mathrm{E}-14$ \\
\hline $\mathrm{Ce} 140$ & $1.77 \mathrm{E}+00$ & $1.76 \mathrm{E}+00$ & $1.74 \mathrm{E}+00$ & $1.74 \mathrm{E}+00$ \\
\hline $\mathrm{Ce} 141$ & $5.63 \mathrm{E}-02$ & $5.58 \mathrm{E}-02$ & $5.53 \mathrm{E}-02$ & $5.53 \mathrm{E}-02$ \\
\hline $\mathrm{Ce} 142$ & $1.57 \mathrm{E}+00$ & $1.56 \mathrm{E}+00$ & $1.55 \mathrm{E}+00$ & $1.55 \mathrm{E}+00$ \\
\hline $\mathrm{Ce} 143$ & $2.18 \mathrm{E}-03$ & $2.15 \mathrm{E}-03$ & $2.12 \mathrm{E}-03$ & $2.12 \mathrm{E}-03$ \\
\hline $\mathrm{Ce} 144$ & $3.71 \mathrm{E}-01$ & $3.67 \mathrm{E}-01$ & $3.63 \mathrm{E}-01$ & $3.64 \mathrm{E}-01$ \\
\hline Cel45 & $1.65 \mathrm{E}-06$ & $1.64 \mathrm{E}-06$ & $1.63 \mathrm{E}-06$ & $1.63 \mathrm{E}-06$ \\
\hline Ce146 & $7.40 \mathrm{E}-06$ & 7.37E-06 & $7.35 \mathrm{E}-06$ & $7.35 \mathrm{E}-06$ \\
\hline $\mathrm{Ce} 147$ & $1.11 \mathrm{E}-07$ & $1.12 \mathrm{E}-07$ & $1.13 \mathrm{E}-07$ & $1.13 \mathrm{E}-07$ \\
\hline $\mathrm{Ce} 148$ & 7.41E-08 & $7.28 \mathrm{E}-08$ & $7.21 \mathrm{E}-08$ & 7.23E-08 \\
\hline Ce149 & 4.67E-13 & $4.61 \mathrm{E}-13$ & $4.55 \mathrm{E}-13$ & $4.57 \mathrm{E}-13$ \\
\hline Ce150 & $1.85 \mathrm{E}-13$ & $1.81 \mathrm{E}-13$ & $1.78 \mathrm{E}-13$ & $1.79 \mathrm{E}-13$ \\
\hline $\operatorname{Pr} 141$ & $1.53 \mathrm{E}+00$ & $1.53 \mathrm{E}+00$ & $1.52 \mathrm{E}+00$ & $1.52 \mathrm{E}+00$ \\
\hline $\operatorname{Pr} 142$ & $3.59 \mathrm{E}-05$ & $3.21 \mathrm{E}-05$ & $2.86 \mathrm{E}-05$ & $2.89 \mathrm{E}-05$ \\
\hline $\operatorname{Pr} 143$ & $2.08 \mathrm{E}-02$ & $2.04 \mathrm{E}-02$ & $2.01 \mathrm{E}-02$ & $2.01 \mathrm{E}-02$ \\
\hline $\operatorname{Pr} 144$ & $1.57 \mathrm{E}-05$ & $1.55 \mathrm{E}-05$ & $1.53 \mathrm{E}-05$ & $1.54 \mathrm{E}-05$ \\
\hline $\operatorname{Pr} 145$ & $2.64 \mathrm{E}-04$ & $2.62 \mathrm{E}-04$ & $2.60 \mathrm{E}-04$ & $2.60 \mathrm{E}-04$ \\
\hline $\operatorname{Pr} 146$ & $1.45 \mathrm{E}-05$ & $1.44 \mathrm{E}-05$ & $1.44 \mathrm{E}-05$ & $1.44 \mathrm{E}-05$ \\
\hline $\operatorname{Pr} 147$ & $6.03 \mathrm{E}-06$ & $6.05 \mathrm{E}-06$ & $6.07 \mathrm{E}-06$ & $6.08 \mathrm{E}-06$ \\
\hline $\operatorname{Pr} 148$ & $6.13 \mathrm{E}-07$ & $6.08 \mathrm{E}-07$ & $6.06 \mathrm{E}-07$ & $6.07 \mathrm{E}-07$ \\
\hline $\operatorname{Pr} 149$ & $3.56 \mathrm{E}-07$ & $3.62 \mathrm{E}-07$ & $3.70 \mathrm{E}-07$ & $3.70 \mathrm{E}-07$ \\
\hline $\operatorname{Pr} 150$ & $6.92 \mathrm{E}-13$ & $6.95 \mathrm{E}-13$ & $7.01 \mathrm{E}-13$ & $7.03 \mathrm{E}-13$ \\
\hline $\operatorname{Pr} 151$ & $6.31 \mathrm{E}-10$ & $6.32 \mathrm{E}-10$ & $6.37 \mathrm{E}-10$ & $6.38 \mathrm{E}-10$ \\
\hline $\operatorname{Pr} 152$ & $1.02 \mathrm{E}-13$ & $1.00 \mathrm{E}-13$ & $9.96 \mathrm{E}-14$ & $1.00 \mathrm{E}-13$ \\
\hline $\operatorname{Pr} 153$ & 4.92E-14 & $4.78 \mathrm{E}-14$ & 4.67E-14 & $4.71 \mathrm{E}-14$ \\
\hline$N d 142$ & $1.90 \mathrm{E}-02$ & $1.74 \mathrm{E}-02$ & $1.59 \mathrm{E}-02$ & $1.60 \mathrm{E}-02$ \\
\hline$N d 143$ & $1.26 \mathrm{E}+00$ & $1.28 \mathrm{E}+00$ & $1.29 \mathrm{E}+00$ & $1.29 \mathrm{E}+00$ \\
\hline$N d 144$ & $1.05 \mathrm{E}+00$ & $1.02 \mathrm{E}+00$ & $9.82 \mathrm{E}-01$ & $9.85 \mathrm{E}-01$ \\
\hline$N d 145$ & $8.81 \mathrm{E}-01$ & $8.84 \mathrm{E}-01$ & 8.87E-01 & $8.87 \mathrm{E}-01$ \\
\hline$N d 146$ & $9.19 \mathrm{E}-01$ & $9.08 \mathrm{E}-01$ & 8.98E-01 & 8.99E-01 \\
\hline$N d 147$ & $7.45 \mathrm{E}-03$ & $7.46 \mathrm{E}-03$ & $7.49 \mathrm{E}-03$ & 7.49E-03 \\
\hline$N d 148$ & $5.34 \mathrm{E}-01$ & $5.35 \mathrm{E}-01$ & $5.36 \mathrm{E}-01$ & $5.37 \mathrm{E}-01$ \\
\hline$N d 149$ & $2.95 \mathrm{E}-05$ & $2.98 \mathrm{E}-05$ & $3.02 \mathrm{E}-05$ & $3.03 \mathrm{E}-05$ \\
\hline$N d 150$ & $3.09 \mathrm{E}-01$ & $3.11 \mathrm{E}-01$ & $3.14 \mathrm{E}-01$ & $3.14 \mathrm{E}-01$ \\
\hline$N d 151$ & $1.88 \mathrm{E}-06$ & $1.92 \mathrm{E}-06$ & $1.96 \mathrm{E}-06$ & $1.97 \mathrm{E}-06$ \\
\hline Nd152 & $1.23 \mathrm{E}-06$ & $1.26 \mathrm{E}-06$ & $1.29 \mathrm{E}-06$ & $1.30 \mathrm{E}-06$ \\
\hline$N d 153$ & $3.59 \mathrm{E}-09$ & 3.63E-09 & $3.76 \mathrm{E}-09$ & $3.78 \mathrm{E}-09$ \\
\hline Nd154 & $9.74 \mathrm{E}-10$ & $9.80 \mathrm{E}-10$ & $9.97 \mathrm{E}-10$ & $1.00 \mathrm{E}-09$ \\
\hline$N d 155$ & $5.55 \mathrm{E}-13$ & $5.49 \mathrm{E}-13$ & $5.48 \mathrm{E}-13$ & $5.52 \mathrm{E}-13$ \\
\hline
\end{tabular}




\begin{tabular}{|c|c|c|c|c|}
\hline $\operatorname{Pm} 147$ & $2.21 \mathrm{E}-01$ & $2.30 \mathrm{E}-01$ & $2.43 \mathrm{E}-01$ & $2.42 \mathrm{E}-01$ \\
\hline Pm148 & $1.18 \mathrm{E}-03$ & $1.14 \mathrm{E}-03$ & $1.07 \mathrm{E}-03$ & $1.08 \mathrm{E}-03$ \\
\hline$P m 148 M$ & $3.86 \mathrm{E}-03$ & $4.29 \mathrm{E}-03$ & $4.66 \mathrm{E}-03$ & $4.65 \mathrm{E}-03$ \\
\hline Pm149 & $1.15 \mathrm{E}-03$ & $1.11 \mathrm{E}-03$ & $1.07 \mathrm{E}-03$ & $1.08 \mathrm{E}-03$ \\
\hline Pm150 & $3.04 \mathrm{E}-07$ & $2.52 \mathrm{E}-07$ & $2.09 \mathrm{E}-07$ & $2.13 \mathrm{E}-07$ \\
\hline Pm151 & 2.82E-04 & $2.88 \mathrm{E}-04$ & $2.95 \mathrm{E}-04$ & $2.95 \mathrm{E}-04$ \\
\hline Pm152 & 4.93E-07 & $5.04 \mathrm{E}-07$ & $5.20 \mathrm{E}-07$ & $5.20 \mathrm{E}-07$ \\
\hline$P m 152 M$ & $1.81 \mathrm{E}-08$ & $2.00 \mathrm{E}-08$ & $2.22 \mathrm{E}-08$ & $2.22 \mathrm{E}-08$ \\
\hline$P m 153$ & $3.80 \mathrm{E}-07$ & $3.88 \mathrm{E}-07$ & $4.02 \mathrm{E}-07$ & $4.05 \mathrm{E}-07$ \\
\hline Pm154 & $9.75 \mathrm{E}-08$ & $9.90 \mathrm{E}-08$ & $1.02 \mathrm{E}-07$ & $1.02 \mathrm{E}-07$ \\
\hline$P m 154 M$ & $5.36 \mathrm{E}-09$ & $5.80 \mathrm{E}-09$ & $6.42 \mathrm{E}-09$ & $6.44 \mathrm{E}-09$ \\
\hline Pm155 & 4.32E-09 & $4.40 \mathrm{E}-09$ & 4.56E-09 & $4.59 \mathrm{E}-09$ \\
\hline Pm156 & $5.67 \mathrm{E}-10$ & $5.76 \mathrm{E}-10$ & $6.02 \mathrm{E}-10$ & $6.05 \mathrm{E}-10$ \\
\hline Pm157 & $1.47 \mathrm{E}-12$ & $1.44 \mathrm{E}-12$ & $1.45 \mathrm{E}-12$ & $1.46 \mathrm{E}-12$ \\
\hline $\mathrm{Sm} 147$ & $1.14 \mathrm{E}-01$ & $1.19 \mathrm{E}-01$ & $1.26 \mathrm{E}-01$ & $1.25 \mathrm{E}-01$ \\
\hline$S m 148$ & $2.41 \mathrm{E}-01$ & $2.39 \mathrm{E}-01$ & $2.32 \mathrm{E}-01$ & $2.33 \mathrm{E}-01$ \\
\hline Sm149 & $9.18 \mathrm{E}-03$ & $1.29 \mathrm{E}-02$ & $1.93 \mathrm{E}-02$ & $1.88 \mathrm{E}-02$ \\
\hline$S m 150$ & $3.96 \mathrm{E}-01$ & $3.89 \mathrm{E}-01$ & $3.80 \mathrm{E}-01$ & $3.81 \mathrm{E}-01$ \\
\hline$S m 151$ & $5.21 \mathrm{E}-02$ & $6.58 \mathrm{E}-02$ & $8.43 \mathrm{E}-02$ & $8.30 \mathrm{E}-02$ \\
\hline Sm152 & $1.83 \mathrm{E}-01$ & $1.87 \mathrm{E}-01$ & $1.93 \mathrm{E}-01$ & $1.93 \mathrm{E}-01$ \\
\hline Sm153 & $1.16 \mathrm{E}-03$ & $1.10 \mathrm{E}-03$ & $1.02 \mathrm{E}-03$ & $1.03 \mathrm{E}-03$ \\
\hline$S m 154$ & 8.77E-02 & 8.83E-02 & 8.98E-02 & $9.02 \mathrm{E}-02$ \\
\hline Sm155 & 8.82E-07 & $9.04 \mathrm{E}-07$ & $9.42 \mathrm{E}-07$ & $9.49 \mathrm{E}-07$ \\
\hline Sm156 & $1.53 \mathrm{E}-05$ & $1.58 \mathrm{E}-05$ & $1.67 \mathrm{E}-05$ & $1.68 \mathrm{E}-05$ \\
\hline Sm157 & $1.31 \mathrm{E}-07$ & $1.33 \mathrm{E}-07$ & $1.38 \mathrm{E}-07$ & $1.40 \mathrm{E}-07$ \\
\hline$S m 158$ & 4.80E-08 & 4.83E-08 & 4.98E-08 & $5.05 \mathrm{E}-08$ \\
\hline Eu151 & $1.82 \mathrm{E}-04$ & $2.91 \mathrm{E}-04$ & 4.77E-04 & 4.61E-04 \\
\hline Eu152 & $1.80 \mathrm{E}-04$ & $2.59 \mathrm{E}-04$ & $3.58 \mathrm{E}-04$ & $3.51 \mathrm{E}-04$ \\
\hline Eu153 & $2.28 \mathrm{E}-01$ & $2.24 \mathrm{E}-01$ & $2.17 \mathrm{E}-01$ & $2.18 \mathrm{E}-01$ \\
\hline Eu154 & 7.33E-02 & $7.28 \mathrm{E}-02$ & $7.02 \mathrm{E}-02$ & 7.07E-02 \\
\hline Eu155 & $1.76 \mathrm{E}-02$ & $1.75 \mathrm{E}-02$ & $1.83 \mathrm{E}-02$ & $1.83 \mathrm{E}-02$ \\
\hline Eu156 & 4.03E-03 & 3.39E-03 & $2.83 \mathrm{E}-03$ & $2.89 \mathrm{E}-03$ \\
\hline Eu157 & $2.05 \mathrm{E}-05$ & $2.01 \mathrm{E}-05$ & $2.03 \mathrm{E}-05$ & $2.06 \mathrm{E}-05$ \\
\hline Eu158 & $5.60 \mathrm{E}-07$ & $5.67 \mathrm{E}-07$ & $5.88 \mathrm{E}-07$ & $5.98 \mathrm{E}-07$ \\
\hline Eu159 & $1.09 \mathrm{E}-07$ & $1.09 \mathrm{E}-07$ & $1.14 \mathrm{E}-07$ & $1.17 \mathrm{E}-07$ \\
\hline Eu160 & $2.90 \mathrm{E}-10$ & $2.89 \mathrm{E}-10$ & $3.07 \mathrm{E}-10$ & $3.18 \mathrm{E}-10$ \\
\hline Gd152 & $2.01 \mathrm{E}-04$ & $2.32 \mathrm{E}-04$ & $2.62 \mathrm{E}-04$ & $2.60 \mathrm{E}-04$ \\
\hline Gd154 & 7.62E-03 & $7.40 \mathrm{E}-03$ & $7.02 \mathrm{E}-03$ & $7.08 \mathrm{E}-03$ \\
\hline Gd155 & $5.96 \mathrm{E}-04$ & $8.13 \mathrm{E}-04$ & $1.22 \mathrm{E}-03$ & $1.19 \mathrm{E}-03$ \\
\hline Gd156 & $1.34 \mathrm{E}-01$ & $1.22 \mathrm{E}-01$ & $1.12 \mathrm{E}-01$ & $1.13 \mathrm{E}-01$ \\
\hline
\end{tabular}




\begin{tabular}{|c|c|c|c|c|}
\hline$G d 157$ & $6.31 \mathrm{E}-04$ & $8.80 \mathrm{E}-04$ & $1.35 \mathrm{E}-03$ & $1.32 \mathrm{E}-03$ \\
\hline$G d 158$ & $5.26 \mathrm{E}-02$ & $5.19 \mathrm{E}-02$ & $5.17 \mathrm{E}-02$ & $5.24 \mathrm{E}-02$ \\
\hline$G d 159$ & $9.53 \mathrm{E}-06$ & $9.44 \mathrm{E}-06$ & $9.65 \mathrm{E}-06$ & $9.87 \mathrm{E}-06$ \\
\hline$G d 160$ & $4.12 \mathrm{E}-03$ & $4.16 \mathrm{E}-03$ & $4.40 \mathrm{E}-03$ & $4.54 \mathrm{E}-03$ \\
\hline$G d 161$ & $4.16 \mathrm{E}-09$ & $4.21 \mathrm{E}-09$ & $4.68 \mathrm{E}-09$ & $4.94 \mathrm{E}-09$ \\
\hline$T b 159$ & $8.54 \mathrm{E}-03$ & $8.63 \mathrm{E}-03$ & $8.95 \mathrm{E}-03$ & $9.10 \mathrm{E}-03$ \\
\hline$T b 160$ & $2.16 \mathrm{E}-04$ & $2.08 \mathrm{E}-04$ & $2.03 \mathrm{E}-04$ & $2.08 \mathrm{E}-04$ \\
\hline$T b 161$ & $1.88 \mathrm{E}-05$ & $1.83 \mathrm{E}-05$ & $1.93 \mathrm{E}-05$ & $2.04 \mathrm{E}-05$ \\
\hline$D y 160$ & $9.85 \mathrm{E}-04$ & $9.71 \mathrm{E}-04$ & $9.74 \mathrm{E}-04$ & $9.94 \mathrm{E}-04$ \\
\hline$D y 161$ & $1.60 \mathrm{E}-03$ & $1.65 \mathrm{E}-03$ & $1.79 \mathrm{E}-03$ & $1.86 \mathrm{E}-03$ \\
\hline$D y 162$ & $7.69 \mathrm{E}-04$ & $7.69 \mathrm{E}-04$ & $8.43 \mathrm{E}-04$ & $8.99 \mathrm{E}-04$ \\
\hline$D y 163$ & $6.95 \mathrm{E}-04$ & $6.83 \mathrm{E}-04$ & $7.39 \mathrm{E}-04$ & $8.10 \mathrm{E}-04$ \\
\hline$D y 164$ & $2.89 \mathrm{E}-04$ & $3.01 \mathrm{E}-04$ & $3.39 \mathrm{E}-04$ & $3.79 \mathrm{E}-04$ \\
\hline$D y 166$ & $8.57 \mathrm{E}-08$ & $8.95 \mathrm{E}-08$ & $1.23 \mathrm{E}-07$ & $1.42 \mathrm{E}-07$ \\
\hline$H o 165$ & $1.62 \mathrm{E}-04$ & $1.35 \mathrm{E}-04$ & $1.24 \mathrm{E}-04$ & $1.43 \mathrm{E}-04$ \\
\hline$E r 166$ & $5.70 \mathrm{E}-05$ & $5.30 \mathrm{E}-05$ & $5.68 \mathrm{E}-05$ & $6.49 \mathrm{E}-05$ \\
\hline$E r 167$ & $4.02 \mathrm{E}-06$ & $4.47 \mathrm{E}-06$ & $6.45 \mathrm{E}-06$ & $7.52 \mathrm{E}-06$ \\
\hline$E r 168$ & $1.15 \mathrm{E}-05$ & $1.15 \mathrm{E}-05$ & $1.38 \mathrm{E}-05$ & $1.65 \mathrm{E}-05$ \\
\hline
\end{tabular}




\section{Appendix 3: Mid-cycle 1-group cross sections of most important actinides}

\begin{tabular}{|c|c|c|c|c|}
\hline Isotope & ThPu & ThPuNp & ThPuNpAm & ThTRU \\
\hline IDs & \multicolumn{4}{|c|}{ Weighted using the total neutron flux } \\
\hline$U 232$ & 11.62000 & 11.09000 & 10.43000 & 10.48000 \\
\hline$U 233$ & 23.97000 & 21.76000 & 18.97000 & 19.14000 \\
\hline$U 234$ & 0.56470 & 0.57340 & 0.58470 & 0.58390 \\
\hline$U 235$ & 12.46000 & 11.08000 & 9.57500 & 9.67700 \\
\hline$U 236$ & 0.35420 & 0.35170 & 0.34890 & 0.34900 \\
\hline$U 238$ & 0.12020 & 0.12240 & 0.12540 & 0.12530 \\
\hline$N p 237$ & 0.62270 & 0.63290 & 0.64600 & 0.64520 \\
\hline Pu238 & 1.84900 & 1.83300 & 1.81600 & 1.81800 \\
\hline Pu239 & 20.77000 & 17.41000 & 13.80000 & 14.04000 \\
\hline Pu240 & 0.64880 & 0.65830 & 0.67100 & 0.67020 \\
\hline Pu241 & 28.04000 & 24.47000 & 20.59000 & 20.84000 \\
\hline Pu242 & 0.50130 & 0.50980 & 0.52090 & 0.52020 \\
\hline Pu244 & 0.43690 & 0.44450 & 0.45460 & 0.45390 \\
\hline Am 241 & 0.79550 & 0.77740 & 0.74730 & 0.74860 \\
\hline$A m 242 m$ & 183.80000 & 158.10000 & 71.38000 & 72.89000 \\
\hline Am243 & 0.49620 & 0.50080 & 0.50490 & 0.50450 \\
\hline $\mathrm{Cm} 242$ & 0.44070 & 0.44120 & 0.44360 & 0.44350 \\
\hline $\mathrm{Cm} 243$ & 41.50000 & 37.90000 & 33.29000 & 33.53000 \\
\hline $\mathrm{Cm} 244$ & 0.87910 & 0.88410 & 0.89110 & 0.88330 \\
\hline $\mathrm{Cm} 245$ & 42.82000 & 38.22000 & 32.91000 & 25.09000 \\
\hline Cm246 & 0.60760 & 0.61220 & 0.61800 & 0.66250 \\
\hline $\mathrm{Cm} 247$ & 15.66000 & 14.65000 & 13.07000 & 18.28000 \\
\hline Cm248 & 0.83000 & 0.82740 & 0.82420 & 0.83220 \\
\hline $\mathrm{Cm} 250$ & 0.54480 & 0.55400 & 0.56610 & 0.66710 \\
\hline$B k 249$ & 0.93880 & 0.87470 & 0.80110 & 0.68440 \\
\hline$C f 249$ & 65.85000 & 58.59000 & 49.53000 & 40.33000 \\
\hline$C f 250$ & 8.92200 & 8.18100 & 7.24200 & 1.13500 \\
\hline$C f 251$ & 176.70000 & 154.70000 & 127.90000 & 88.60000 \\
\hline$C f 252$ & 4.35000 & 4.18500 & 3.96600 & 3.96300 \\
\hline
\end{tabular}




\begin{tabular}{|c|c|c|c|c|}
\hline Isotope & ThPu & ThPuNp & ThPuNpAm & ThTRU \\
\hline IDs & \multicolumn{4}{|c|}{ Weighted using the total neutron flux } \\
\hline$U 232$ & 5.36500 & 4.99300 & 4.56300 & 4.58900 \\
\hline U233 & 3.93000 & 3.62500 & 3.21000 & 3.23200 \\
\hline$U 234$ & 17.55000 & 16.62000 & 14.97000 & 15.05000 \\
\hline$U 235$ & 4.65700 & 4.33000 & 3.94900 & 3.97400 \\
\hline$U 236$ & 8.95700 & 8.40200 & 7.71000 & 7.74400 \\
\hline$U 238$ & 7.21200 & 6.92800 & 6.49500 & 6.51800 \\
\hline Np237 & 19.90000 & 17.39000 & 14.97000 & 15.11000 \\
\hline Pu238 & 8.05000 & 7.02200 & 5.91600 & 5.99500 \\
\hline Pu239 & 11.43000 & 9.55300 & 7.53300 & 7.66900 \\
\hline Pu240 & 24.41000 & 20.54000 & 15.84000 & 16.16000 \\
\hline Pu241 & 9.12300 & 7.83700 & 6.43800 & 6.53700 \\
\hline Pu242 & 13.25000 & 11.66000 & 9.74800 & 9.90300 \\
\hline Pu244 & 2.83500 & 2.77400 & 2.69100 & 2.69900 \\
\hline$A m 241$ & 37.11000 & 32.22000 & 25.04000 & 25.40000 \\
\hline$A m 242 m$ & 40.50000 & 34.58000 & 12.97000 & 13.25000 \\
\hline$A m 243$ & 39.50000 & 35.29000 & 26.64000 & 26.92000 \\
\hline $\mathrm{Cm} 242$ & 3.30000 & 3.18100 & 3.02400 & 3.03700 \\
\hline $\mathrm{Cm} 243$ & 6.19300 & 5.65600 & 5.00100 & 5.03800 \\
\hline $\mathrm{Cm} 244$ & 15.47000 & 14.57000 & 13.44000 & 10.89000 \\
\hline $\mathrm{Cm} 245$ & 7.15800 & 6.38400 & 5.49500 & 3.71500 \\
\hline $\mathrm{Cm} 246$ & 2.85100 & 2.74100 & 2.59200 & 2.92300 \\
\hline $\mathrm{Cm} 247$ & 9.86700 & 8.99800 & 7.87400 & 8.46400 \\
\hline $\mathrm{Cm} 248$ & 6.95200 & 6.70800 & 6.39700 & 5.92300 \\
\hline $\mathrm{Cm} 250$ & 50.28000 & 47.81000 & 43.86000 & 7.61900 \\
\hline$B k 249$ & 100.80000 & 88.14000 & 73.43000 & 46.34000 \\
\hline$C f 249$ & 23.29000 & 20.72000 & 17.47000 & 10.06000 \\
\hline$C f 250$ & 236.90000 & 211.80000 & 175.90000 & 140.80000 \\
\hline$C f 251$ & 73.95000 & 64.14000 & 52.54000 & 34.36000 \\
\hline$C f 252$ & 1.73800 & 1.66100 & 1.56700 & 1.40100 \\
\hline
\end{tabular}

